DESY $16-148$

DO-TH 16/15

September 2016

\title{
The Asymptotic 3-Loop Heavy Flavor Corrections to the Charged Current Structure Functions
}

$$
F_{L}^{W^{+}-W^{-}}\left(x, Q^{2}\right) \text { and } F_{2}^{W^{+}-W^{-}}\left(x, Q^{2}\right)
$$

\author{
A. Behring ${ }^{a}$, J. Blümlein ${ }^{a}$, G. Falcioni ${ }^{a}$, A. De Freitas ${ }^{a}$, A. von Manteuffel ${ }^{b}$, \\ and C. Schneider ${ }^{c}$ \\ ${ }^{a}$ Deutsches Elektronen-Synchrotron, DESY, \\ Platanenallee 6, D-15738 Zeuthen, Germany \\ ${ }^{b}$ Department of Physics and Astronomy, Michigan State University, \\ East Lansing, MI 48824, USA, \\ PRISMA Cluster of Excellence, Johannes Gutenberg University, 55099 Mainz, Germany \\ ${ }^{c}$ Research Institute for Symbolic Computation (RISC), \\ Johannes Kepler University, Altenbergerstraße 69, A-4040 Linz, Austria
}

\begin{abstract}
We calculate the massive Wilson coefficients for the heavy flavor contributions to the non-singlet charged current deep-inelastic scattering structure functions $F_{L}^{W^{+}}\left(x, Q^{2}\right)-$ $F_{L}^{W^{-}}\left(x, Q^{2}\right)$ and $F_{2}^{W^{+}}\left(x, Q^{2}\right)-F_{2}^{W^{-}}\left(x, Q^{2}\right)$ in the asymptotic region $Q^{2} \gg m^{2}$ to 3-loop order in Quantum Chromodynamics (QCD) at general values of the Mellin variable $N$ and the momentum fraction $x$. Besides the heavy quark pair production, also the single heavy flavor excitation $s \rightarrow c$ contributes. Numerical results are presented for the charm quark contributions and consequences on the unpolarized Bjorken sum rule and Adler sum rule are discussed.
\end{abstract}




\section{Introduction}

The flavor non-singlet charged current structure functions $F_{1,2}^{W^{+}-W^{-}}\left(x, Q^{2}\right)$ can be measured in deep-inelastic neutrino(antineutrino)-nucleon scattering and in high energy charged leptonnucleon scattering in $e p$ or $\mu p$ collisions. They are associated with the well-known unpolarized Bjorken sum rule [1] and Adler sum rule [2] by their first moment, the former of which can be used for QCD tests measuring the strong coupling constant $a_{s}=\alpha_{s} /(4 \pi)=g_{s}^{2} /(4 \pi)^{2}$. These structure functions also allow for an associated determination of the valence quark distributions of the nucleon. The massless contributions to these combinations of structure functions have been calculated recently to 3 -loop order [3]. In the present paper we compute the asymptotic heavy flavor corrections to these flavor non-singlet structure functions in the region $Q^{2} \gg m^{2}$ to the same order, with $m$ the heavy quark mass and $Q^{2}$ the virtuality of the process, and present numerical results in the case of charm quark contributions.

The massless and massive QCD corrections at first order in the coupling constant have been computed in Refs. [4 $7 \sqrt{1}$ and in Refs. $9 \sqrt{13}$ to $O\left(a_{s}^{2}\right)^{2}$. The massive $O\left(a_{s}^{2}\right)$ corrections were calculated in the asymptotic representation [14], which is valid at high scales $Q^{2}$. To obtain an estimate of the range of validity, one may perform an $O\left(a_{s}\right)$ comparison with the complete result for the process of single heavy quark excitation [6,7]. Likewise, a comparison is possible for the $O\left(a_{s}^{2}\right)$ corrections, which were given in complete form in Ref. [15] for the Wilson coefficient with the gauge boson coupling to the massless fermions and assuming an approximation for the Cabibbo-suppressed flavor excitation term $s^{\prime} \rightarrow c$, where the additional charm quark in the final state has been dealt with as being massless.

The charged current scattering cross sections are given by 16, 17

$$
\begin{aligned}
\frac{d \sigma^{\nu(\bar{\nu})}}{d x d y}= & \frac{G_{F}^{2} s}{4 \pi} \frac{M_{W}^{4}}{\left(M_{W}^{2}+Q^{2}\right)^{2}} \\
& \times\left\{\left(1+(1-y)^{2}\right) F_{2}^{W^{ \pm}}\left(x, Q^{2}\right)-y^{2} F_{L}^{W^{ \pm}}\left(x, Q^{2}\right) \pm\left(1-(1-y)^{2}\right) x F_{3}^{W^{ \pm}}\left(x, Q^{2}\right)\right\} \\
\frac{d \sigma^{l(\bar{l})}}{d x d y}= & \frac{G_{F}^{2} s}{4 \pi} \frac{M_{W}^{4}}{\left(M_{W}^{2}+Q^{2}\right)^{2}} \\
& \times\left\{\left(1+(1-y)^{2}\right) F_{2}^{W^{\mp}}\left(x, Q^{2}\right)-y^{2} F_{L}^{W^{\mp}}\left(x, Q^{2}\right) \pm\left(1-(1-y)^{2}\right) x F_{3}^{W^{\mp}}\left(x, Q^{2}\right)\right\}
\end{aligned}
$$

where $x=Q^{2} / y s$ and $y=q . P / l . P$ denote the Bjorken variables, $l$ and $P$ are the incoming lepton and nucleon 4-momenta, and $s=(l+P)^{2} . G_{F}$ is the Fermi constant and $M_{W}$ the mass of the $W$-boson. $F_{i}^{W^{ \pm}}\left(x, Q^{2}\right)$ are the structure functions, where the $+(-)$ signs refer to incoming neutrinos (antineutrinos) and charged antileptons (leptons), respectively. We will consider the combination of structure functions

$$
F_{1,2}^{W^{+-} W^{-}}\left(x, Q^{2}\right)=F_{1,2}^{W^{+}}\left(x, Q^{2}\right)-F_{1,2}^{W^{-}}\left(x, Q^{2}\right)
$$

in the following. The longitudinal structure function is obtained by

$$
F_{L}\left(x, Q^{2}\right)=F_{2}\left(x, Q^{2}\right)-2 x F_{1}\left(x, Q^{2}\right) .
$$

\footnotetext{
${ }^{1}$ The massive 1-loop corrections given in 8 were corrected in 7 , see also 6 .

${ }^{2}$ Some results given in 9 have been corrected in Ref. 12 .
} 
The combinations $(1.3)$ can be measured projecting onto the kinematic factor $Y_{+}=1+(1-y)^{2}$ in the case of $F_{2}$ for the differential cross sections at $x, Q^{2}=$ const. and by varying $s$ in addition, in the case of $F_{L}$.

The main formalism to obtain the massive Wilson coefficients in the asymptotic range $Q^{2} \gg m^{2}$, i.e. $L_{q, L, 2}^{W^{+}-W^{-}, \mathrm{NS}}$ and $H_{q, L, 2}^{W^{+}-W^{-}, \mathrm{NS}}$, has been outlined in Refs. 12, 14, 18. They are composed of the massive non-singlet operator matrix elements (OMEs) [19] and the massless Wilson coefficients [3] up to 3-loop order. The following representation of the structure functions is obtained

$$
\begin{aligned}
F_{L, 2}^{W^{+}-W^{-}}\left(x, Q^{2}\right)= & 2 x\left\{[ | V _ { d u } | ^ { 2 } ( d - \overline { d } ) + | V _ { s u } | ^ { 2 } ( s - \overline { s } ) - V _ { u } ( u - \overline { u } ) ] \otimes \left[C_{q, L, 2}^{W^{+}-W^{-}, \mathrm{NS}}\right.\right. \\
& \left.\left.+L_{q, L, 2}^{W^{+}-W^{-}, \mathrm{NS}}\right]+\left[\left|V_{d c}\right|^{2}(d-\bar{d})+\left|V_{s c}\right|^{2}(s-\bar{s})\right] \otimes H_{q, L, 2}^{W^{+}-W^{-}, \mathrm{NS}}\right\},
\end{aligned}
$$

with one massless Wilson coefficient $C_{q, L, 2}^{W^{+}-W^{-}, \mathrm{NS}}$ and two massive Wilson coefficients $L_{q, L, 2}^{W^{+}-W^{-}, \mathrm{NS}}$, $H_{q, L, 2}^{W^{+}-W^{-}, \mathrm{NS}}$, see Sections 2 and 3 . The coefficients $V_{i j}$ are the Cabibbo-Kobayashi-Maskawa (CKM) 20, 21 matrix elements, where $V_{u}=\left|V_{d u}\right|^{2}+\left|V_{s u}\right|^{2}$, and the present numerical values are 22

$$
\left|V_{d u}\right|=0.97425, \quad\left|V_{s u}\right|=0.2253, \quad\left|V_{d c}\right|=0.225, \quad\left|V_{s c}\right|=0.986
$$

with

$$
\begin{aligned}
u-\bar{u} & =u_{v}, \\
d-\bar{d} & =d_{v}, \\
s-\bar{s} & \approx 0 .
\end{aligned}
$$

In the following we will consider only the charm quark corrections with $m \equiv m_{c}$ the charm quark mass in the on-shell scheme. The transformation to the $\overline{\mathrm{MS}}$ scheme has been given in Ref. [19]. We note that the 3-loop asymptotic charm quark corrections to the combination of structure functions $x F_{3}^{W^{+}+W^{-}}\left(x, Q^{2}\right)$ have been calculated in Ref. 23 and related corrections to the twist-2 contributions of the polarized structure functions $g_{1,2}\left(x, Q^{2}\right)$ in Ref. [24].

A series of asymptotic 3-loop heavy flavor Wilson coefficients have also been calculated for neutral current scattering along with the transition matrix elements in the variable flavor number scheme, see Refs. [25] for recent surveys.

\section{The Structure Function $F_{L}\left(x, Q^{2}\right)$}

The massive Wilson coefficients depend on the logarithms

$$
L_{M}=\ln \left(\frac{m^{2}}{\mu^{2}}\right), \quad L_{Q}=\ln \left(\frac{Q^{2}}{\mu^{2}}\right) .
$$

Here $\mu$ denotes the factorization scale. For the Wilson coefficients in Mellin $N$ space we consider the following series in the strong coupling constant

$$
L_{q, 2(L)}^{W^{+}-W^{-}, \mathrm{NS}}=\delta_{2,0}+\sum_{k=1}^{\infty} a_{s}^{k} L_{q, 2(L)}^{W^{+}-W^{-}, \mathrm{NS},(k)},
$$




$$
\begin{aligned}
& H_{q, 2(L)}^{W^{+}-W^{-}, \mathrm{NS}}=\delta_{2,0}+\sum_{k=1}^{\infty} a_{s}^{k} H_{q, 2(L)}^{W^{+-} W^{-}, \mathrm{NS},(k)} \\
& C_{q, 2(L)}^{W^{+}-W^{-}, \mathrm{NS}}=\delta_{2,0}+\sum_{k=1}^{\infty} a_{s}^{k} C_{q, 2(L)}^{W^{+}-W^{-}, \mathrm{NS},(k)}
\end{aligned}
$$

In the following we drop the arguments of the nested harmonic sums [26] and harmonic polylogarithms [27] by defining $S_{\vec{a}}(N) \equiv S_{\vec{a}}$ and $H_{\vec{b}}(x) \equiv H_{\vec{b}}$. by

The 3-loop contributions to the Wilson coefficient $L_{q, L}^{W^{+}-W^{-}, \mathrm{NS},(3)}$ in Mellin $N$ space are given

$$
\begin{aligned}
& L_{q, L}^{W^{+}-W^{-}, \mathrm{NS},(3)}= \\
& C_{F} T_{F}^{2}\left(2 N_{F}+1\right)\left[L_{Q}^{2} \frac{64}{9(N+1)}-L_{Q}\left(\frac{64\left(19 N^{2}+7 N-6\right)}{27 N(N+1)^{2}}+\frac{128 S_{1}}{9(N+1)}\right)\right] \\
& +C_{A} C_{F} T_{F}\left[-L_{Q}^{2} \frac{352}{9(N+1)}+L_{Q}\left(\frac{1088}{9(N+1)} S_{1}+\frac{128}{3(N+1)} S_{3}+\frac{128}{3(N+1)} S_{-3}\right.\right. \\
& +\left(-\frac{256\left(N^{2}+N+1\right)}{3(N-1)(N+1)(N+2)}+\frac{256}{3(N+1)} S_{1}\right) S_{-2}-\frac{256}{3(N+1)} S_{-2,1} \\
& \left.\left.+\frac{16 P_{4}}{27(N-1) N^{2}(N+1)^{3}(N+2)}-\frac{128}{N+1} \zeta_{3}\right)\right] \\
& +C_{F}^{2} T_{F}\left[L_{Q}^{2}\left(\frac{8\left(3 N^{2}+3 N+2\right)}{N(N+1)^{2}}-\frac{32}{N+1} S_{1}\right)+L_{M}^{2}\left(\frac{8\left(3 N^{2}+3 N+2\right)}{3 N(N+1)^{2}}\right.\right. \\
& \left.-\frac{32}{3(N+1)} S_{1}\right)+L_{M}\left(\frac{8 P_{1}}{9 N^{2}(N+1)^{3}}-\frac{320}{9(N+1)} S_{1}+\frac{64}{3(N+1)} S_{2}\right) \\
& +L_{Q}\left(\frac{128}{3(N+1)} S_{1}^{2}-\frac{32 P_{2}}{9(N-1) N^{2}(N+1)^{3}(N+2)}-\frac{16(N+10)(5 N+3)}{9 N(N+1)^{2}} S_{1}\right. \\
& -\frac{256}{3(N+1)} S_{3}+\left(\frac{512\left(N^{2}+N+1\right)}{3(N-1)(N+1)(N+2)}-\frac{512}{3(N+1)} S_{1}\right) S_{-2} \\
& \left.-\frac{128}{3(N+1)} S_{2}-\frac{256}{3(N+1)} S_{-3}+\frac{512}{3(N+1)} S_{-2,1}+\frac{256}{N+1} \zeta_{3}\right) \\
& \left.+\frac{2 P_{3}}{27 N^{3}(N+1)^{4}}-\frac{896}{27(N+1)} S_{1}+\frac{160}{9(N+1)} S_{2}-\frac{32}{3(N+1)} S_{3}\right]+\hat{c}_{q, L}^{(3)},
\end{aligned}
$$

where $\hat{c}_{q, L}^{(3)}=c_{q, L}^{(3)}\left(N_{F}+1\right)-c_{q, L}^{(3)}\left(N_{F}\right)$ is the 3-loop massless contribution, cf. [3]. The color factors in the case of QCD are $C_{A}=N_{c}=3, C_{F}=\left(N_{c}^{2}-1\right) /\left(2 N_{c}\right), T_{F}=1 / 2, N_{c}=3$ and $N_{F}$ denotes the number of massless flavors. Except for $\hat{c}_{q, L}^{(3)}\left(N_{F}\right)$, the Wilson coefficient is expressed by harmonic sums up to weight $\mathrm{w}=3$. The polynomials $P_{i}$ above read

$$
\begin{aligned}
& P_{1}=3 N^{4}+6 N^{3}+47 N^{2}+20 N-12 \\
& P_{2}=36 N^{6}+81 N^{5}-125 N^{4}-319 N^{3}-211 N^{2}-14 N-24 \\
& P_{3}=219 N^{6}+657 N^{5}+1193 N^{4}+763 N^{3}-40 N^{2}-48 N+72 \\
& P_{4}=469 N^{6}+1143 N^{5}-515 N^{4}-2055 N^{3}-746 N^{2}+120 N-144 .
\end{aligned}
$$


By performing a Mellin inversion, the corresponding representation in $x$ space is obtained, which reads

$$
\begin{aligned}
& L_{q, L}^{W^{+}-W^{-}, \mathrm{NS},(3)}= \\
& C_{A} C_{F} T_{F}\left[-L_{Q}^{2} \frac{352 x}{9}+L_{Q}\left(\frac{16}{27}(781 x-312)+\left(\frac{128\left(2 x^{3}+x^{2}-1\right) H_{0}}{3 x}\right.\right.\right. \\
& \left.+\frac{64}{3} x H_{0}^{2}\right) H_{-1}+\frac{832}{9} x H_{0}-\frac{128}{3} x H_{-1}^{2} H_{0}+\left(\frac{1088 x}{9}+\frac{64}{3} x H_{0}^{2}\right) H_{1} \\
& -\frac{128}{3} x^{2} H_{0}^{2}+\left(-\frac{128\left(2 x^{3}+x^{2}-1\right)}{3 x}+\frac{256}{3} x H_{-1}+\frac{128}{3} x H_{0}\right) H_{0,-1} \\
& -\frac{128}{3} x H_{0} H_{0,1}-\frac{256}{3} x H_{0,-1,-1}-128 x H_{0,0,-1}+\frac{128}{3} x H_{0,0,1}+\left(\frac{256 x^{2}}{3}\right. \\
& \left.\left.\left.-\frac{128}{3} x H_{-1}-\frac{128}{3} x H_{1}\right) \zeta_{2}\right)\right] \\
& +C_{F} T_{F}^{2} N_{F}\left[L_{Q}^{2} \frac{128 x}{9}-L_{Q}\left(\frac{128}{27}(25 x-6)+\frac{512}{9} x H_{0}+\frac{256}{9} x H_{1}\right)\right] \\
& +C_{F} T_{F}^{2}\left[L_{Q}^{2} \frac{64 x}{9}-L_{Q}\left(\frac{64}{27}(25 x-6)+\frac{256}{9} x H_{0}+\frac{128}{9} x H_{1}\right)\right] \\
& +C_{F}^{2} T_{F}\left[L_{Q}^{2}\left(8(x+2)-16 x H_{0}-32 x H_{1}\right)+L_{M}^{2}\left(\frac{8(x+2)}{3}-\frac{16}{3} x H_{0}\right.\right. \\
& \left.-\frac{32}{3} x H_{1}\right)+L_{M}\left(\frac{32}{9}(x+3) H_{0}-\frac{8}{9}(53 x-56)-\frac{16}{3} x H_{0}^{2}+\frac{64}{3} x H_{0,1}\right. \\
& \left.+\left(-\frac{320 x}{9}-\frac{64}{3} x H_{0}\right) H_{1}\right)+L_{Q}\left(-\frac{32}{9}(83 x-47)+\left(\frac{256}{3} x H_{1}+\frac{256}{3} x H_{-1}\right.\right. \\
& \left.-\frac{32}{3} x(16 x+11)\right) \zeta_{2}-\left(\frac{256\left(2 x^{3}+x^{2}-1\right) H_{0}}{3 x}+\frac{128}{3} x H_{0}^{2}\right) H_{-1} \\
& +\frac{256}{3} x H_{-1}^{2} H_{0}+\frac{16}{9}(89 x-24) H_{0}+\frac{16}{3} x(16 x+7) H_{0}^{2}+\left(\frac{80}{9}(5 x-6)\right. \\
& \left.+\frac{256}{3} x H_{0}-\frac{128}{3} x H_{0}^{2}\right) H_{1}+\frac{128}{3} x H_{1}^{2}+\left(\frac{256\left(2 x^{3}+x^{2}-1\right)}{3 x}-\frac{512}{3} x H_{-1}\right. \\
& \left.-\frac{256}{3} x H_{0}\right) H_{0,-1}+\left(32 x+\frac{256}{3} x H_{0}\right) H_{0,1}+\frac{512}{3} x H_{0,-1,-1}+256 x H_{0,0,-1} \\
& \left.-\frac{256}{3} x H_{0,0,1}\right)-\frac{2}{27}(653 x-872)+\frac{16}{27}(11 x+42) H_{0}+\frac{8}{9}(x+3) H_{0}^{2} \\
& -\frac{8}{9} x H_{0}^{3}+\left(-\frac{896 x}{27}-\frac{160}{9} x H_{0}-\frac{16}{3} x H_{0}^{2}\right) H_{1}+\left(\frac{160 x}{9}+\frac{32}{3} x H_{0}\right) H_{0,1} \\
& \left.-\frac{32}{3} x H_{0,0,1}\right]+\hat{c}_{q, L}^{(3)} \text {. }
\end{aligned}
$$

Here $\zeta_{k}, k \in \mathbb{N}, k \geq 2$, are the values of the Riemann $\zeta$ function at integer argument. Except 
for $\hat{c}_{q, L}^{(3)}\left(N_{F}\right)$, the Wilson coefficient is expressed by weighted harmonic polylogarithms of up to weight $\mathrm{w}=3$.

The contribution of the massive Wilson coefficient $H_{q, L}$ is found by combining the massless Wilson coefficient $C_{q, L}$ and $L_{q, L}$ :

$$
H_{q, L}\left(x, Q^{2}\right)=C_{q, L}\left(N_{F}, x, Q^{2}\right)+L_{q, L}\left(x, Q^{2}\right) .
$$

Eq. (1.4) provides the relation to the Wilson coefficients of the structure function $F_{1}\left(x, Q^{2}\right)$.

\section{The Structure Function $F_{2}\left(x, Q^{2}\right)$}

The asymptotic massive 3-loop Wilson coefficient $L_{q, 2}^{W^{+}-W^{-}, \mathrm{NS},(3)}$ in Mellin $N$ space reads

$$
\begin{aligned}
& L_{q, 2}^{W^{+}-W^{-}, \mathrm{NS},(3)}= \\
& C_{F} T_{F}^{2}\left\{-\frac{2008\left(3 N^{2}+3 N+2\right)}{243 N(N+1)}+L_{M}^{3}\left(\frac{8\left(3 N^{2}+3 N+2\right)}{9 N(N+1)}-\frac{32}{9} S_{1}\right)\right. \\
& +L_{M}^{2}\left(\frac{8 P_{1}}{27 N^{2}(N+1)^{2}}-\frac{320}{27} S_{1}+\frac{64}{9} S_{2}\right)+L_{M}\left(\frac{2 P_{3}}{81 N^{3}(N+1)^{3}}-\frac{896}{81} S_{1}\right. \\
& \left.+\frac{160}{27} S_{2}-\frac{32}{9} S_{3}\right)+\left(\frac{8032}{243}-\frac{128 \zeta_{3}}{3}\right) S_{1}+\frac{32\left(3 N^{2}+3 N+2\right) \zeta_{3}}{3 N(N+1)} \\
& +\left(N_{F}+\frac{1}{2}\right)\left[\frac{4 P_{41}}{729 N^{4}(N+1)^{4}}+L_{Q}^{3}\left(\frac{32\left(3 N^{2}+3 N+2\right)}{27 N(N+1)}-\frac{128}{27} S_{1}\right)\right. \\
& +L_{M}^{3}\left(\frac{16\left(3 N^{2}+3 N+2\right)}{27 N(N+1)}-\frac{64}{27} S_{1}\right)+L_{Q}^{2}\left(\frac{64}{9} S_{1}^{2}-\frac{64}{3} S_{2}-\frac{16 P_{11}}{27 N^{2}(N+1)^{2}}\right. \\
& \left.+\frac{32\left(29 N^{2}+29 N-6\right) S_{1}}{27 N(N+1)}\right)+L_{M}\left(\frac{4 P_{33}}{81 N^{3}(N+1)^{3}}-\frac{2176}{81} S_{1}-\frac{320}{27} S_{2}\right. \\
& \left.+\frac{64}{9} S_{3}\right)+L_{Q}\left(\frac{16 P_{34}}{81 N^{3}(N+1)^{3}}+\left(-\frac{32 P_{15}}{81 N^{2}(N+1)^{2}}+\frac{128}{9} S_{2}\right) S_{1}-\frac{128}{27} S_{1}^{3}\right. \\
& \left.-\frac{32\left(29 N^{2}+29 N-6\right) S_{1}^{2}}{27 N(N+1)}+\frac{32\left(35 N^{2}+35 N-2\right) S_{2}}{9 N(N+1)}-\frac{1792}{27} S_{3}+\frac{256}{9} S_{2,1}\right) \\
& \left.\left.+\left(\frac{512 \zeta_{3}}{27}-\frac{24064}{729}\right) S_{1}+\frac{128}{81} S_{2}+\frac{640}{81} S_{3}-\frac{128}{27} S_{4}-\frac{128\left(3 N^{2}+3 N+2\right) \zeta_{3}}{27 N(N+1)}\right]\right\} \\
& +C_{F}^{2} T_{F}\left\{\frac{P_{47}}{162 N^{5}(N+1)^{5}}-\frac{S_{-2,1}}{N^{2}(N+1)} \frac{128}{81}\left(112 N^{3}+112 N^{2}-39 N+18\right)\right. \\
& +L_{Q}^{3}\left(\frac{1}{N^{2}(N+1)^{2}} \frac{2}{3}\left(3 N^{2}+3 N+2\right)^{2}-\frac{16\left(3 N^{2}+3 N+2\right) S_{1}}{3 N(N+1)}+\frac{32}{3} S_{1}^{2}\right) \\
& +L_{Q}^{2}\left(-\frac{2 P_{31}}{9 N^{3}(N+1)^{3}}+\left(\frac{2 P_{16}}{9 N^{2}(N+1)^{2}}+\frac{176}{3} S_{2}\right) S_{1}-16 S_{1}^{3}+\frac{64}{3} S_{-3}+\frac{64}{3} S_{3}\right. \\
& -\frac{4\left(107 N^{2}+107 N-54\right) S_{1}^{2}}{9 N(N+1)}-\frac{44\left(3 N^{2}+3 N+2\right) S_{2}}{3 N(N+1)}-\frac{128}{3} S_{-2,1}+\left(\frac{128}{3} S_{1}\right. \\
& \left.\left.-\frac{64}{3 N(N+1)}\right) S_{-2}\right)+L_{M}^{2}\left(L _ { Q } \left(\frac{1}{N^{2}(N+1)^{2}} \frac{2}{3}\left(3 N^{2}+3 N+2\right)^{2}\right.\right.
\end{aligned}
$$




$$
\begin{aligned}
& \left.-\frac{16\left(3 N^{2}+3 N+2\right) S_{1}}{3 N(N+1)}+\frac{32}{3} S_{1}^{2}\right)-\frac{2(N-1) P_{25}}{3 N^{3}(N+1)^{3}}+\left(\frac{2 P_{9}}{3 N^{2}(N+1)^{2}}+\frac{80}{3} S_{2}\right) S_{1} \\
& -\frac{4(N-1)(N+2) S_{1}^{2}}{N(N+1)}-\frac{16}{3} S_{1}^{3}-\frac{20\left(3 N^{2}+3 N+2\right) S_{2}}{3 N(N+1)}+\frac{64}{3} S_{3}+\left(\frac{128}{3} S_{1}\right. \\
& \left.\left.-\frac{64}{3 N(N+1)}\right) S_{-2}+\frac{64}{3} S_{-3}-\frac{128}{3} S_{-2,1}\right)+L_{M}\left(L _ { Q } \left(\left(-\frac{8 P_{10}}{9 N^{2}(N+1)^{2}}\right.\right.\right. \\
& \left.-\frac{64}{3} S_{2}\right) S_{1}+\frac{1}{N^{3}(N+1)^{3}} \frac{2}{9}\left(3 N^{2}+3 N+2\right) P_{1}+\frac{320}{9} S_{1}^{2} \\
& \left.+\frac{16\left(3 N^{2}+3 N+2\right) S_{2}}{3 N(N+1)}\right)+\frac{P_{40}}{9 N^{4}(N+1)^{4}}-\left(\frac{256}{3} S_{3}+\frac{256}{3} S_{-2,1}-64 \zeta_{3}\right. \\
& \left.-\frac{2 P_{32}}{9 N^{3}(N+1)^{3}}-\frac{16\left(59 N^{2}+59 N-6\right) S_{2}}{9 N(N+1)}\right) S_{1}+\left(\frac{32}{3} S_{2}-\frac{4 P_{7}}{3 N^{2}(N+1)^{2}}\right) S_{1}^{2} \\
& -\frac{160}{9} S_{1}^{3}-\frac{4 P_{13} S_{2}}{9 N^{2}(N+1)^{2}}-32 S_{2}^{2}+\frac{32\left(29 N^{2}+29 N+12\right) S_{3}}{9 N(N+1)}-\frac{256}{3} S_{4} \\
& +\left(-\frac{64\left(16 N^{2}+10 N-3\right)}{9 N^{2}(N+1)^{2}}+\frac{1280}{9} S_{1}-\frac{128}{3} S_{2}\right) S_{-2}-\frac{128}{3} S_{-4}+\frac{128}{3} S_{3,1} \\
& +\left(\frac{64\left(10 N^{2}+10 N+3\right)}{9 N(N+1)}-\frac{128}{3} S_{1}\right) S_{-3}-\frac{128\left(10 N^{2}+10 N-3\right) S_{-2,1}}{9 N(N+1)} \\
& \left.-\frac{128}{3} S_{-2,2}+\frac{512}{3} S_{-2,1,1}-\frac{16\left(3 N^{2}+3 N+2\right) \zeta_{3}}{N(N+1)}\right)+L_{Q}\left[\frac{4 P_{44}}{27 N^{4}(N+1)^{4}(N+2)}\right. \\
& +\left(-\frac{4 P_{37}}{27 N^{3}(N+1)^{3}}+\frac{640}{9} S_{3}+\frac{64}{3} S_{2,1}-\frac{32\left(67 N^{2}+67 N-21\right) S_{2}}{9 N(N+1)}+\frac{512}{3} S_{-2,1}\right. \\
& \left.+64 \zeta_{3}\right) S_{1}+\left(\frac{2 P_{19}}{27 N^{2}(N+1)^{2}}-\frac{224}{3} S_{2}\right) S_{1}^{2}+\frac{32(4 N-1)(4 N+5) S_{1}^{3}}{9 N(N+1)}+\frac{80}{9} S_{1}^{4} \\
& +\frac{2 P_{18} S_{2}}{9 N^{2}(N+1)^{2}}+48 S_{2}^{2}-\frac{32\left(53 N^{2}+77 N+4\right) S_{3}}{9 N(N+1)}+\frac{352}{3} S_{4}+64 S_{-2}^{2}+\frac{448}{3} S_{-4} \\
& +\left(-\frac{64 P_{23}}{9 N^{2}(N+1)^{2}(N+2)}-\frac{128\left(10 N^{2}+22 N-9\right) S_{1}}{9 N(N+1)}-\frac{256}{3} S_{1}^{2}+\frac{256}{3} S_{2}\right) S_{-2} \\
& +\left(\frac{256}{3} S_{1}-\frac{64\left(10 N^{2}+22 N+3\right)}{9 N(N+1)}\right) S_{-3}+64 S_{3,1}+\frac{16\left(9 N^{2}+9 N-2\right) S_{2,1}}{3 N(N+1)} \\
& +\frac{128\left(10 N^{2}+22 N-9\right) S_{-2,1}}{9 N(N+1)}-\frac{256}{3} S_{-3,1}-64 S_{2,1,1}-\frac{512}{3} S_{-2,1,1} \\
& \left.-\frac{16\left(9 N^{2}-7 N+6\right) \zeta_{3}}{N(N+1)}\right]-\frac{1}{N(N+1)} \frac{48}{5}\left(3 N^{2}+3 N+2\right) \zeta_{2}^{2}+\left(\frac{P_{42}}{162 N^{4}(N+1)^{4}}\right. \\
& +\frac{8 P_{20} S_{2}}{81 N^{2}(N+1)^{2}}-\frac{64}{9} S_{2}^{2}-\frac{8\left(347 N^{2}+347 N+54\right) S_{3}}{27 N(N+1)}+\frac{704}{9} S_{4}+\frac{128 S_{2,1}}{9 N(N+1)} \\
& -\frac{320}{9} S_{3,1}-\frac{256\left(10 N^{2}+10 N-3\right) S_{-2,1}}{27 N(N+1)}-\frac{256}{9} S_{-2,2}+\frac{64}{3} S_{2,1,1}+\frac{1024}{9} S_{-2,1,1} \\
& \left.+\frac{192 \zeta_{2}^{2}}{5}-\frac{1208 \zeta_{3}}{9}\right) S_{1}+\left(\frac{P_{29}}{9 N^{3}(N+1)^{3}}+\frac{16\left(5 N^{2}+5 N-4\right) S_{2}}{9 N(N+1)}+16 S_{3}\right.
\end{aligned}
$$




$$
\begin{aligned}
& \left.-\frac{128}{9} S_{2,1}-\frac{256}{9} S_{-2,1}\right) S_{1}^{2}+\left(-\frac{16 P_{8}}{27 N^{2}(N+1)^{2}}+\frac{128}{27} S_{2}\right) S_{1}^{3}+\frac{512}{9} S_{5} \\
& +\left(\frac{P_{28}}{81 N^{3}(N+1)^{3}}+\frac{400}{27} S_{3}+\frac{256}{3} S_{-2,1}-\frac{64 \zeta_{3}}{3}\right) S_{2}+\frac{8 P_{21} S_{3}}{81 N^{2}(N+1)^{2}} \\
& -\frac{32\left(23 N^{2}+23 N-3\right) S_{2}^{2}}{27 N(N+1)}-\frac{176\left(17 N^{2}+17 N+6\right) S_{4}}{27 N(N+1)}+\left(-\frac{64 P_{14}}{81 N^{3}(N+1)^{3}}\right. \\
& \left.+\frac{128 P_{12} S_{1}}{81 N^{2}(N+1)^{2}}-\frac{128 S_{1}^{2}}{9 N(N+1)}+\frac{256}{27} S_{1}^{3}-\frac{1280}{27} S_{2}+\frac{512}{27} S_{3}-\frac{512}{9} S_{2,1}\right) S_{-2} \\
& +\left(\frac{1}{N(N+1)^{2}} \frac{64}{81}\left(112 N^{3}+224 N^{2}+169 N+39\right)+\frac{128}{9} S_{1}^{2}+\frac{128}{9} S_{2}\right. \\
& \left.-\frac{128\left(10 N^{2}+10 N+3\right) S_{1}}{27 N(N+1)}\right) S_{-3}+\left(-\frac{128\left(10 N^{2}+10 N+3\right)}{27 N(N+1)}+\frac{256}{9} S_{1}\right) S_{-4} \\
& +\frac{256}{9} S_{-5}+\frac{16 P_{5} S_{2,1}}{9 N^{2}(N+1)^{2}}+\frac{256}{9} S_{2,3}-\frac{512}{9} S_{2,-3}-\frac{512}{9} S_{4,1}+\frac{512}{9} S_{-2,3} \\
& +\frac{16\left(89 N^{2}+89 N+30\right) S_{3,1}}{27 N(N+1)}-\frac{128\left(10 N^{2}+10 N-3\right) S_{-2,2}}{27 N(N+1)}+\frac{512}{9} S_{2,1,-2} \\
& -\frac{16\left(3 N^{2}+3 N+2\right) S_{2,1,1}}{3 N(N+1)}+\frac{256}{9} S_{3,1,1}+\frac{512\left(10 N^{2}+10 N-3\right) S_{-2,1,1}}{27 N(N+1)} \\
& \left.+\frac{512}{9} S_{-2,2,1}-\frac{2048}{9} S_{-2,1,1,1}+\frac{2 P_{17} \zeta_{3}}{9 N^{2}(N+1)^{2}}\right\} \\
& +C_{A} C_{F} T_{F}\left\{\frac{P_{46}}{729 N^{5}(N+1)^{5}}+\frac{S_{-2,1}}{N^{2}(N+1)} \frac{64}{81}\left(112 N^{3}+112 N^{2}-39 N+18\right)\right. \\
& +L_{M}^{3}\left(-\frac{44\left(3 N^{2}+3 N+2\right)}{27 N(N+1)}+\frac{176}{27} S_{1}\right)+L_{Q}^{3}\left(-\frac{88\left(3 N^{2}+3 N+2\right)}{27 N(N+1)}\right. \\
& \left.+\frac{352}{27} S_{1}\right)+L_{M}^{2}\left(\frac{1}{N^{3}(N+1)^{3}} \frac{2}{9}\left(3 N^{2}+3 N+2\right) P_{6}-\frac{184}{9} S_{1}-\frac{32}{3} S_{3}-\frac{32}{3} S_{-3}\right. \\
& \left.+\left(\frac{32}{3 N(N+1)}-\frac{64}{3} S_{1}\right) S_{-2}+\frac{64}{3} S_{-2,1}\right)+L_{Q}^{2}\left(-\frac{176}{9} S_{1}^{2}+\frac{176}{3} S_{2}-\frac{32}{3} S_{-3}\right. \\
& -\frac{32}{3} S_{3}+\frac{2 P_{36}}{27 N^{3}(N+1)^{3}}-\frac{16\left(194 N^{2}+194 N-33\right) S_{1}}{27 N(N+1)}+\frac{64}{3} S_{-2,1} \\
& \left.+\left(\frac{32}{3 N(N+1)}-\frac{64}{3} S_{1}\right) S_{-2}\right)+L_{Q}\left(-\frac{4 P_{45}}{81 N^{4}(N+1)^{4}(N+2)}+\frac{352}{27} S_{1}^{3}\right. \\
& -\frac{S_{2}}{N(N+1)^{2}} \frac{16}{9}\left(230 N^{3}+460 N^{2}+213 N-11\right)+\left(\frac{4 P_{38}}{81 N^{3}(N+1)^{3}}+32 S_{3}\right. \\
& \left.-\frac{32\left(11 N^{2}+11 N+3\right) S_{2}}{9 N(N+1)}-\frac{128}{3} S_{2,1}-\frac{256}{3} S_{-2,1}-64 \zeta_{3}\right) S_{1}+\left(\frac{32}{3} S_{2}\right. \\
& \left.+\frac{16\left(194 N^{2}+194 N-33\right)}{27 N(N+1)}\right) S_{1}^{2}-\frac{32}{3} S_{2}^{2}+\frac{16\left(368 N^{2}+440 N-45\right) S_{3}}{27 N(N+1)}-\frac{224}{3} S_{4} \\
& +\left(\frac{32 P_{23}}{9 N^{2}(N+1)^{2}(N+2)}+\frac{64\left(10 N^{2}+22 N-9\right) S_{1}}{9 N(N+1)}+\frac{128}{3} S_{1}^{2}-\frac{128}{3} S_{2}\right) S_{-2}
\end{aligned}
$$




$$
\begin{aligned}
& -32 S_{-2}^{2}+\left(\frac{32\left(10 N^{2}+22 N+3\right)}{9 N(N+1)}-\frac{128}{3} S_{1}\right) S_{-3}-\frac{224}{3} S_{-4}+\frac{128}{3} S_{-3,1}-\frac{64}{3} S_{3,1} \\
& -\frac{64\left(11 N^{2}+11 N-3\right) S_{2,1}}{9 N(N+1)}-\frac{64\left(10 N^{2}+22 N-9\right) S_{-2,1}}{9 N(N+1)}+64 S_{2,1,1} \\
& \left.+\frac{256}{3} S_{-2,1,1}+\frac{32\left(3 N^{2}-N+2\right) \zeta_{3}}{N(N+1)}\right)+L_{M}\left(\frac{P_{39}}{81 N^{4}(N+1)^{4}}+\frac{1792}{27} S_{2}\right. \\
& +\left(-\frac{8 P_{30}}{81 N^{3}(N+1)^{3}}+32 S_{3}+\frac{128}{3} S_{-2,1}-64 \zeta_{3}\right) S_{1}-\frac{16\left(31 N^{2}+31 N+9\right) S_{3}}{9 N(N+1)} \\
& +\frac{160}{3} S_{4}+\left(\frac{32\left(16 N^{2}+10 N-3\right)}{9 N^{2}(N+1)^{2}}-\frac{640}{9} S_{1}+\frac{64}{3} S_{2}\right) S_{-2}-\frac{128}{3} S_{3,1}+\frac{64}{3} S_{-4} \\
& +\left(-\frac{32\left(10 N^{2}+10 N+3\right)}{9 N(N+1)}+\frac{64}{3} S_{1}\right) S_{-3}+\frac{64\left(10 N^{2}+10 N-3\right) S_{-2,1}}{9 N(N+1)} \\
& \left.+\frac{64}{3} S_{-2,2}-\frac{256}{3} S_{-2,1,1}+\frac{16\left(3 N^{2}+3 N+2\right) \zeta_{3}}{N(N+1)}\right)+\left(\frac{4 P_{43}}{729 N^{4}(N+1)^{4}}\right. \\
& -\frac{S_{2}}{N^{2}(N+1)^{2}} \frac{16}{9}(N-1)\left(2 N^{3}-N^{2}-N-2\right)+\frac{112}{9} S_{2}^{2}+\frac{80(2 N+1)^{2} S_{3}}{9 N(N+1)} \\
& +\frac{64}{3} S_{3,1}-\frac{208}{9} S_{4}-\frac{8\left(9 N^{2}+9 N+16\right) S_{2,1}}{9 N(N+1)}+\frac{128\left(10 N^{2}+10 N-3\right) S_{-2,1}}{27 N(N+1)} \\
& \left.+\frac{128}{9} S_{-2,2}-32 S_{2,1,1}-\frac{512}{9} S_{-2,1,1}-\frac{192 \zeta_{2}^{2}}{5}+\frac{4\left(593 N^{2}+593 N+108\right) \zeta_{3}}{27 N(N+1)}\right) S_{1} \\
& +\frac{1}{N(N+1)} \frac{48}{5}\left(3 N^{2}+3 N+2\right) \zeta_{2}^{2}+\left(\frac{4 P_{22}}{9 N^{3}(N+1)^{3}}+\frac{32 S_{2}}{9 N(N+1)}-\frac{80}{9} S_{3}\right. \\
& \left.+\frac{128}{9} S_{2,1}+\frac{128}{9} S_{-2,1}-16 \zeta_{3}\right) S_{1}^{2}+\left(\frac{4 P_{35}}{81 N^{3}(N+1)^{3}}+\frac{496}{27} S_{3}-\frac{64}{3} S_{2,1}\right. \\
& \left.-\frac{128}{3} S_{-2,1}+16 \zeta_{3}\right) S_{2}-\frac{64}{27} S_{1}^{3} S_{2}-\frac{4\left(15 N^{2}+15 N+14\right) S_{2}^{2}}{9 N(N+1)}-\frac{8 P_{26} S_{3}}{81 N^{2}(N+1)^{2}} \\
& +\frac{4\left(443 N^{2}+443 N+78\right) S_{4}}{27 N(N+1)}-\frac{224}{9} S_{5}+\left(\frac{32 P_{14}}{81 N^{3}(N+1)^{3}}+\frac{64 S_{1}^{2}}{9 N(N+1)}\right. \\
& \left.-\frac{64 P_{12} S_{1}}{81 N^{2}(N+1)^{2}}-\frac{128}{27} S_{1}^{3}+\frac{640}{27} S_{2}-\frac{256}{27} S_{3}+\frac{256}{9} S_{2,1}\right) S_{-2}-\left(\frac{64}{9} S_{1}^{2}+\frac{64}{9} S_{2}\right. \\
& \left.+\frac{32}{81} \frac{\left(112 N^{3}+224 N^{2}+169 N+39\right)}{N(N+1)^{2}}-\frac{64\left(10 N^{2}+10 N+3\right) S_{1}}{27 N(N+1)}\right) S_{-3}-\frac{128}{9} S_{-5} \\
& +\left(\frac{64\left(10 N^{2}+10 N+3\right)}{27 N(N+1)}-\frac{128}{9} S_{1}\right) S_{-4}-\frac{128}{3} S_{2,3}-\frac{8 P_{24} S_{2,1}}{9 N^{2}(N+1)^{2}}+\frac{256}{9} S_{2,-3} \\
& -\frac{8(13 N+4)(13 N+9) S_{3,1}}{27 N(N+1)}+\frac{256}{9} S_{4,1}+\frac{64\left(10 N^{2}+10 N-3\right) S_{-2,2}}{27 N(N+1)}+\frac{64}{3} S_{2,2,1} \\
& -\frac{256}{9} S_{-2,3}+\frac{8\left(3 N^{2}+3 N+2\right) S_{2,1,1}}{N(N+1)}-\frac{256}{9} S_{2,1,-2}-\frac{256}{9} S_{3,1,1}-\frac{256}{9} S_{-2,2,1} \\
& \left.-\frac{256\left(10 N^{2}+10 N-3\right) S_{-2,1,1}}{27 N(N+1)}+\frac{224}{9} S_{2,1,1,1}+\frac{1024}{9} S_{-2,1,1,1}+\frac{P_{27} \zeta_{3}}{27 N^{2}(N+1)^{2}}\right\} \\
& +\hat{c}_{q, 2}^{(3)}
\end{aligned}
$$


where $\hat{c}_{q, 2}^{(3)}=c_{q, 2}^{(3)}\left(N_{F}+1\right)-c_{q, 2}^{(3)}\left(N_{F}\right)$ is obtained from the 3-loop massless Wilson coefficient Ref. 3]. Except for $\hat{c}_{q, 2}^{(3)}\left(N_{F}\right)$, the Wilson coefficient is expressed by harmonic sums up to weight $\mathbf{w}=5$. The polynomials in the equation above are defined as follows

$$
\begin{aligned}
& P_{5}=7 N^{4}+14 N^{3}+3 N^{2}-4 N-4 \\
& P_{6}=17 N^{4}+34 N^{3}+29 N^{2}+12 N+24 \\
& P_{7}=19 N^{4}+38 N^{3}-9 N^{2}-20 N+4 \\
& P_{8}=28 N^{4}+56 N^{3}+28 N^{2}+2 N+1 \\
& P_{9}=33 N^{4}+38 N^{3}-15 N^{2}-60 N-28 \\
& P_{10}=33 N^{4}+66 N^{3}+97 N^{2}+40 N-12 \\
& P_{11}=57 N^{4}+72 N^{3}+29 N^{2}-22 N-24 \\
& P_{12}=112 N^{4}+224 N^{3}+121 N^{2}+9 N+9 \\
& P_{13}=141 N^{4}+198 N^{3}+169 N^{2}-32 N-84 \\
& P_{14}=181 N^{4}+266 N^{3}+82 N^{2}-3 N+18 \\
& P_{15}=235 N^{4}+596 N^{3}+319 N^{2}+66 N+72 \\
& P_{16}=501 N^{4}+750 N^{3}+325 N^{2}-188 N-204 \\
& P_{17}=561 N^{4}+1122 N^{3}+767 N^{2}+302 N+48 \\
& P_{18}=1131 N^{4}+1926 N^{3}+1019 N^{2}-64 N-276 \\
& P_{19}=1139 N^{4}+3286 N^{3}+1499 N^{2}+504 N+828 \\
& P_{20}=1199 N^{4}+2398 N^{3}+1181 N^{2}+18 N+90 \\
& P_{21}=1220 N^{4}+2251 N^{3}+1772 N^{2}+303 N-138 \\
& P_{22}=3 N^{5}+11 N^{4}+10 N^{3}+19 N^{2}+23 N+16 \\
& P_{23}=6 N^{5}-25 N^{3}-45 N^{2}-11 N+6 \\
& P_{24}=12 N^{5}+16 N^{4}+18 N^{3}-15 N^{2}-5 N-8 \\
& P_{25}=15 N^{5}+39 N^{4}+39 N^{3}-17 N^{2}-32 N-20 \\
& P_{26}=27 N^{5}+863 N^{4}+1573 N^{3}+1151 N^{2}+144 N-36 \\
& P_{27}=648 N^{5}-2103 N^{4}-4278 N^{3}-3505 N^{2}-682 N-432 \\
& P_{28}=-11145 N^{6}-30915 N^{5}-33923 N^{4}-11449 N^{3}+1960 N^{2}-1032 N-2088 \\
& P_{29}=-151 N^{6}-469 N^{5}-181 N^{4}+305 N^{3}+80 N^{2}-88 N-56 \\
& P_{30}=155 N^{6}+465 N^{5}+465 N^{4}+155 N^{3}+108 N^{2}+108 N+54 \\
& P_{31}=216 N^{6}+459 N^{5}+417 N^{4}-3 N^{3}-125 N^{2}-80 N+12 \\
& P_{32}=309 N^{6}+647 N^{5}+293 N^{4}-783 N^{3}-718 N^{2}+68 N+216 \\
& P_{33}=525 N^{6}+1575 N^{5}+1535 N^{4}+973 N^{3}+536 N^{2}+48 N-72 \\
& P_{34}=609 N^{6}+1029 N^{5}+613 N^{4}-37 N^{3}-74 N^{2}+300 N+216 \\
& P_{35}=868 N^{6}+2469 N^{5}+2487 N^{4}+940 N^{3}+171 N^{2}+207 N+144 \\
& P_{36}=1407 N^{6}+3297 N^{5}+2891 N^{4}+727 N^{3}-514 N^{2}-240 N+144 \\
& P_{37}=1770 N^{6}+4731 N^{5}+4483 N^{4}+749 N^{3}+55 N^{2}+1440 N+756 \\
& P_{38}=7531 N^{6}+26121 N^{5}+27447 N^{4}+8815 N^{3}+1110 N^{2}+936 N-324 \\
& P_{39}=-4785 N^{8}-19140 N^{7}-18754 N^{6}+1320 N^{5}+12723 N^{4}+6548 N^{3}+4080 N^{2} \\
& -648 N-1728 \\
& P_{40}=-45 N^{8}-138 N^{7}-774 N^{6}-476 N^{5}-881 N^{4}-762 N^{3}-868 N^{2}-88 N+192
\end{aligned}
$$




$$
\begin{aligned}
P_{41}= & 3549 N^{8}+14196 N^{7}+23870 N^{6}+25380 N^{5}+15165 N^{4}+1712 N^{3}-2016 N^{2} \\
& +144 N+432 \\
P_{42}= & -3456 B_{4} N^{4}(N+1)^{4}+42591 N^{8}+161388 N^{7}+226848 N^{6}+105790 N^{5} \\
& -26735 N^{4}-28666 N^{3}+3560 N^{2}-3192 N-4464 \\
P_{43}= & 1944 B_{4} N^{4}(N+1)^{4}-10807 N^{8}-43228 N^{7}-63222 N^{6}-40150 N^{5}-14587 N^{4} \\
& -9018 N^{3}-7452 N^{2}-2376 N-324 \\
P_{44}= & 828 N^{9}+3456 N^{8}+4539 N^{7}+2412 N^{6}+1852 N^{5}+5026 N^{4}+4703 N^{3}+2468 N^{2} \\
& -324 N-576 \\
P_{45}= & 8274 N^{9}+39795 N^{8}+71627 N^{7}+64189 N^{6}+29919 N^{5}+8096 N^{4}+5620 N^{3} \\
& +5664 N^{2}-1368 N-2160 \\
P_{46}= & -1944 B_{4} N^{4}(N+1)^{4}\left(3 N^{2}+3 N+2\right)+165 N^{10}+825 N^{9}+109664 N^{8}+331682 N^{7} \\
& +457641 N^{6}+346145 N^{5}+219290 N^{4}+86724 N^{3}+13608 N^{2}+14256 N+10368 \\
P_{47}= & 864 B_{4} N^{4}(N+1)^{4}\left(3 N^{2}+3 N+2\right)-18351 N^{10}-87156 N^{9}-198195 N^{8}-244182 N^{7} \\
& -184797 N^{6}-70160 N^{5}-23209 N^{4}-8030 N^{3}-984 N^{2}-2328 N-2160 .
\end{aligned}
$$

Here the constant $B_{4}$ is given by

$$
B_{4}=-4 \zeta_{2} \ln ^{2}(2)+\frac{2}{3} \ln ^{4}(2)-\frac{13}{2} \zeta_{4}+16 \operatorname{Li}_{4}\left(\frac{1}{2}\right) .
$$

By performing the Mellin inversion to $x$-space one obtains

$$
\begin{aligned}
L_{q, 2}^{W^{+}-} W^{-}, \mathrm{NS},(3) & = \\
& \delta(1-x)\left\{C _ { A } C _ { F } T _ { F } \left[-\frac{44 L_{M}^{3}}{9}-\frac{88 L_{Q}^{3}}{9}+L_{M}^{2}\left(\frac{34}{3}-\frac{16 \zeta_{3}}{3}\right)+L_{Q}^{2}\left(\frac{938}{9}\right.\right.\right. \\
& \left.-\frac{16 \zeta_{3}}{3}\right)+L_{M}\left(-\frac{1595}{27}+\frac{136 \zeta_{2}^{2}}{15}+\frac{272 \zeta_{3}}{9}\right)+L_{Q}\left(-\frac{11032}{27}-\frac{32 \zeta_{2}}{3}-\frac{392 \zeta_{2}^{2}}{15}\right. \\
& \left.\left.+\frac{1024 \zeta_{3}}{9}\right)+\frac{5248 \zeta_{2}^{2}}{135}-\frac{10045 \zeta_{3}}{81}-\frac{16}{9} \zeta_{2} \zeta_{3}-\frac{176 \zeta_{5}}{9}+\frac{55}{243}-8 B_{4}\right] \\
+ & C_{F}^{2} T_{F}\left[6 L_{M}^{2} L_{Q}+6 L_{Q}^{3}+L_{M}^{2}\left(\frac{32 \zeta_{3}}{3}-10\right)+2 L_{M} L_{Q}+L_{Q}^{2}\left(\frac{32 \zeta_{3}}{3}-48\right)\right. \\
& -L_{M}\left(5+\frac{272 \zeta_{2}^{2}}{15}+\frac{112 \zeta_{3}}{9}\right)+L_{Q}\left(\frac{368}{3}+\frac{64 \zeta_{2}}{3}+\frac{784 \zeta_{2}^{2}}{15}-\frac{1616 \zeta_{3}}{9}\right)-\frac{6608 \zeta_{2}^{2}}{135} \\
+ & \left.\frac{13682 \zeta_{3}}{81}+\frac{32 \zeta_{2} \zeta_{3}}{9}+\frac{352 \zeta_{5}}{9}-\frac{2039}{18}+16 B_{4}\right]+C_{F} N_{F} T_{F}^{2}\left[\frac{16 L_{M}^{3}}{9}+\frac{32 L_{Q}^{3}}{9}\right. \\
& \left.-\frac{304 L_{Q}^{2}}{9}+\frac{700 L_{M}}{27}+\frac{3248 L_{Q}}{27}-\frac{128 \zeta_{3}}{9}+\frac{4732}{243}\right]+C_{F} T_{F}^{2}\left[\frac{32 L_{M}^{3}}{9}+\frac{16 L_{Q}^{3}}{9}\right. \\
+ & \left.\left.\frac{8 L_{M}^{2}}{9}-\frac{152 L_{Q}^{2}}{9}+\frac{496 L_{M}}{27}+\frac{1624 L_{Q}}{27}+\frac{224 \zeta_{3}}{9}-\frac{3658}{243}\right]\right\} \\
+ & \left\{\frac { C _ { F } N _ { F } T _ { F } ^ { 2 } } { 1 - x } \left[\frac{64 L_{M}^{3}}{27}+\frac{128 L_{Q}^{3}}{27}-L_{Q}^{2}\left(\frac{928}{27}+\frac{256}{9} H_{0}+\frac{128}{9} H_{1}\right)+L_{M}\left(\frac{2176}{81}\right.\right.\right. \\
& \left.-\frac{320}{27} H_{0}-\frac{32}{9} H_{0}^{2}\right)+L_{Q}\left(\frac{7520}{81}+\frac{4288 H_{0}}{27}+\frac{128}{3} H_{0}^{2}+\frac{1856 H_{1}}{27}+\frac{256}{9} H_{0} H_{1}\right. \\
&
\end{aligned}
$$




$$
\begin{aligned}
& \left.\left.+\frac{128}{9} H_{1}^{2}+\frac{256}{9} H_{0,1}-\frac{512 \zeta_{2}}{9}\right)+\frac{128 H_{0}}{81}-\frac{320}{81} H_{0}^{2}-\frac{64}{81} H_{0}^{3}-\frac{512 \zeta_{3}}{27}+\frac{24064}{729}\right] \\
& +\frac{C_{F} T_{F}^{2}}{1-x}\left[\frac{128 L_{M}^{3}}{27}+\frac{64 L_{Q}^{3}}{27}+L_{M}^{2}\left(\frac{320}{27}+\frac{64 H_{0}}{9}\right)-L_{Q}^{2}\left(\frac{464}{27}+\frac{128}{9} H_{0}\right.\right. \\
& \left.+\frac{64}{9} H_{1}\right)+\frac{1984 L_{M}}{81}+L_{Q}\left(\frac{3760}{81}+\frac{2144 H_{0}}{27}+\frac{64}{3} H_{0}^{2}+\frac{928 H_{1}}{27}+\frac{128}{9} H_{0} H_{1}\right. \\
& \left.\left.+\frac{64}{9} H_{1}^{2}+\frac{128}{9} H_{0,1}-\frac{256 \zeta_{2}}{9}\right)+\frac{64 H_{0}}{81}-\frac{160}{81} H_{0}^{2}-\frac{32}{81} H_{0}^{3}+\frac{896 \zeta_{3}}{27}-\frac{12064}{729}\right] \\
& +\frac{C_{A} C_{F} T_{F}}{(1-x)^{2}}\left[\frac{32}{9}(x+2) H_{0,1}-\frac{4}{81}(800 x-773) H_{0}^{2}+\frac{32}{81}(94 x-121) \zeta_{2}\right] \\
& +\frac{C_{A} C_{F} T_{F}}{1-x}\left[-\frac{176 L_{M}^{3}}{27}-\frac{352 L_{Q}^{3}}{27}+L_{Q}^{2}\left(\frac{3104}{27}+\frac{704 H_{0}}{9}+\frac{16}{3} H_{0}^{2}+\frac{352 H_{1}}{9}-\frac{32 \zeta_{2}}{3}\right)\right. \\
& +L_{M}^{2}\left(\frac{184}{9}+\frac{16}{3} H_{0}^{2}-\frac{32 \zeta_{2}}{3}\right)+L_{Q}\left(-\frac{30124}{81}-\frac{14144}{27} H_{0}-\frac{1216}{9} H_{0}^{2}-\frac{80}{9} H_{0}^{3}\right. \\
& -\frac{6208}{27} H_{1}-\frac{704}{9} H_{0} H_{1}-\frac{16}{3} H_{0}^{2} H_{1}-\frac{352}{9} H_{1}^{2}+\frac{32}{3} H_{0} H_{1}^{2}-64 H_{0} H_{0,-1} \\
& -\frac{704}{9} H_{0,1}+\frac{32}{3} H_{0} H_{0,1}-\frac{128}{3} H_{1} H_{0,1}+128 H_{0,0,-1}-\frac{128}{3} H_{0,0,1}+64 H_{0,1,1} \\
& \left.+\left(192+\frac{128 H_{0}}{3}+64 H_{1}\right) \zeta_{2}-\frac{256 \zeta_{3}}{3}\right)+L_{M}\left(\frac{1240}{81}+\frac{1792 H_{0}}{27}+\frac{248}{9} H_{0}^{2}\right. \\
& \left.+\frac{32}{9} H_{0}^{3}-16 H_{0}^{2} H_{1}+32 H_{0} H_{0,1}-\frac{64}{3} H_{0,0,1}+\left(-\frac{320}{9}-\frac{64}{3} H_{0}\right) \zeta_{2}+96 \zeta_{3}\right) \\
& +\left(-\frac{496}{27} H_{0}-\frac{112}{9} H_{0}^{2}+8 H_{1}-\frac{160}{9} H_{0} H_{1}-\frac{128}{9} H_{1}^{2}+\frac{32}{9} H_{0,1}\right) \zeta_{2}+\frac{43228}{729} \\
& -\frac{32 B_{4}}{3}+\frac{3256 H_{0}}{81}+\frac{496}{81} H_{0}^{3}+\frac{16}{27} H_{0}^{4}+\frac{32}{3} H_{1}-\frac{32}{9} H_{0} H_{1}-\frac{160}{9} H_{0}^{2} H_{1} \\
& -\frac{112}{27} H_{0}^{3} H_{1}+\frac{8}{9} H_{0}^{2} H_{1}^{2}-\frac{64}{27} H_{0} H_{1}^{3}+\frac{368}{9} H_{0} H_{0,1}+\frac{16}{3} H_{0}^{2} H_{0,1}-8 H_{1} H_{0,1} \\
& -\frac{128}{9} H_{0} H_{1} H_{0,1}+\frac{128}{9} H_{1}^{2} H_{0,1}-\frac{32}{9} H_{0,1}^{2}-\frac{1072}{27} H_{0,0,1}+\frac{32}{9} H_{0} H_{0,0,1} \\
& +\frac{320}{9} H_{1} H_{0,0,1}+24 H_{0,1,1}+\frac{160}{9} H_{0} H_{0,1,1}-32 H_{1} H_{0,1,1}-\frac{224}{9} H_{0,0,1,1} \\
& \left.+\frac{224}{9} H_{0,1,1,1}+\frac{592 \zeta_{2}^{2}}{15}+\left(-\frac{1196}{27}+\frac{160 H_{0}}{9}-\frac{32}{9} H_{1}\right) \zeta_{3}\right] \\
& +\frac{C_{F}^{2} T_{F}}{1-x}\left[L_{M}^{2} L_{Q}\left(16-\frac{32}{3} H_{0}-\frac{64}{3} H_{1}\right)+L_{Q}^{3}\left(16-\frac{32}{3} H_{0}-\frac{64}{3} H_{1}\right)-L_{M}^{2}(22\right. \\
& \left.+16 H_{0}-\frac{16}{3} H_{0}^{2}-8 H_{1}-\frac{128}{3} H_{0} H_{1}-16 H_{1}^{2}+\frac{64 \zeta_{2}}{3}\right)+L_{Q}^{2}\left(-\frac{334}{3}+\frac{32 H_{0}}{9}\right. \\
& \left.+\frac{80}{3} H_{0}^{2}+\frac{856 H_{1}}{9}+\frac{320}{3} H_{0} H_{1}+48 H_{1}^{2}-\frac{256 \zeta_{2}}{3}\right)+L_{M} L_{Q}\left(\frac{88}{3}-\frac{176}{9} H_{0}\right. \\
& \left.-\frac{32}{3} H_{0}^{2}-\frac{640}{9} H_{1}-\frac{64}{3} H_{0} H_{1}+\frac{64 \zeta_{2}}{3}\right)+L_{M}\left(\frac{88}{9} H_{0}^{2}-\frac{206}{3}-\frac{112}{3} H_{0}+\frac{64}{9} H_{0}^{3}\right. \\
& +\frac{152 H_{1}}{3}+\frac{1424}{9} H_{0} H_{1}+\frac{160}{3} H_{0}^{2} H_{1}+\frac{160}{3} H_{1}^{2}+\frac{32}{3} H_{0} H_{1}^{2}-\frac{64}{3} H_{0} H_{0,1}-\left(\frac{784}{9}\right.
\end{aligned}
$$




$$
\begin{aligned}
& \left.\left.+\frac{128}{3} H_{0}+\frac{64}{3} H_{1}\right) \zeta_{2}-64 \zeta_{3}\right)+L_{Q}\left(\frac{2360}{9}+\frac{4508 H_{0}}{27}-\frac{160}{3} H_{0}^{2}-\frac{224}{9} H_{0}^{3}\right. \\
& -\frac{4556}{27} H_{1}-\frac{3680}{9} H_{0} H_{1}-128 H_{0}^{2} H_{1}-\frac{512}{3} H_{1}^{2}-128 H_{0} H_{1}^{2}-\frac{320}{9} H_{1}^{3} \\
& +128 H_{0} H_{0,-1}+48 H_{0,1}-\frac{64}{3} H_{0} H_{0,1}+\frac{64}{3} H_{1} H_{0,1}-256 H_{0,0,-1}-64 H_{0,1,1} \\
& \left.+\left(\frac{2608}{9}+\frac{832 H_{0}}{3}+\frac{448 H_{1}}{3}\right) \zeta_{2}+320 \zeta_{3}\right)+\frac{64 B_{4}}{3}-\left(\frac{2488}{27}+\frac{1192}{27} H_{0}+\frac{80}{9} H_{0}^{2}\right. \\
& \left.+\frac{160}{9} H_{1}-\frac{32}{3} H_{0} H_{1}-\frac{128}{9} H_{1}^{2}+\frac{32}{9} H_{0,1}\right) \zeta_{2}-\frac{3262}{27} H_{0}+\frac{196}{27} H_{0}^{2}+\frac{380}{81} H_{0}^{3} \\
& +\frac{4}{3} H_{0}^{4}+\frac{302 H_{1}}{9}+\frac{13624}{81} H_{0} H_{1}+\frac{1628}{27} H_{0}^{2} H_{1}+\frac{304}{27} H_{0}^{3} H_{1}+\frac{448}{9} H_{1}^{2} \\
& +\frac{80}{9} H_{0} H_{1}^{2}-\frac{8}{9} H_{0}^{2} H_{1}^{2}+\frac{128}{27} H_{0} H_{1}^{3}+\frac{112}{9} H_{0,1}-\frac{1304}{27} H_{0} H_{0,1}-\frac{32}{3} H_{0}^{2} H_{0,1} \\
& +\frac{160}{9} H_{0} H_{1} H_{0,1}-\frac{128}{9} H_{1}^{2} H_{0,1}-\frac{16}{9} H_{0,1}^{2}+\frac{1184}{27} H_{0,0,1}+\frac{128}{9} H_{0} H_{0,0,1} \\
& -\frac{256}{9} H_{1} H_{0,0,1}-16 H_{0,1,1}-\frac{32}{3} H_{0} H_{0,1,1}+\frac{64}{3} H_{1} H_{0,1,1}-\frac{128}{9} H_{0,0,0,1} \\
& \left.\left.+\frac{64}{3} H_{0,0,1,1}-\frac{1328 \zeta_{2}^{2}}{45}+\left(\frac{3088}{27}-\frac{128}{9} H_{0}+\frac{160 H_{1}}{9}\right) \zeta_{3}-\frac{14197}{54}\right]\right\}_{+} \\
& +\left\{C _ { F } ^ { 2 } T _ { F } \left[L_{M}^{2} L_{Q}\left(8(x+1) H_{0}+\frac{32}{3}(x+1) H_{1}-\frac{8}{3}(x+5)\right)\right.\right. \\
& +L_{Q}^{3}\left(8(x+1) H_{0}+\frac{32}{3}(x+1) H_{1}-\frac{8}{3}(x+5)\right)+L_{M} L_{Q}\left(\frac{4}{9}(19 x-85)\right. \\
& +\frac{8}{3}(13 x+1) H_{0}+8(x+1) H_{0}^{2}+\frac{128}{9}(4 x+1) H_{1}+\frac{32}{3}(x+1) H_{0} H_{1} \\
& \left.-\frac{32}{3}(x+1) \zeta_{2}\right)+L_{M}^{2}\left(12(5 x-2)-16(2 x+1) H_{0}+\frac{64\left(x^{2}+1\right) H_{-1} H_{0}}{3(x+1)}\right. \\
& -\frac{4\left(9 x^{2}+10 x+9\right) H_{0}^{2}}{3(x+1)}-\frac{16}{3}(3 x+2) H_{1}-\frac{64}{3}(x+1) H_{0} H_{1}-8(x+1) H_{1}^{2} \\
& \left.-\frac{64\left(x^{2}+1\right) H_{0,-1}}{3(x+1)}-\frac{8}{3}(x+1) H_{0,1}+\frac{8\left(9 x^{2}+10 x+9\right) \zeta_{2}}{3(x+1)}\right) \\
& +L_{Q}^{2}\left(\frac{4}{9}(188 x+157)-\frac{88}{3}(3 x+1) H_{0}+\frac{64\left(x^{2}+1\right) H_{-1} H_{0}}{3(x+1)}-24(x+1) H_{1}^{2}\right. \\
& -\frac{16}{9}(59 x+26) H_{1}-\frac{4\left(21 x^{2}+34 x+21\right) H_{0}^{2}}{3(x+1)}-\frac{160}{3}(x+1) H_{0} H_{1} \\
& \left.-8(x+1) H_{0,1}-\frac{64\left(x^{2}+1\right) H_{0,-1}}{3(x+1)}+\frac{8\left(23 x^{2}+38 x+23\right) \zeta_{2}}{3(x+1)}\right) \\
& +L_{M}\left(\frac{4}{3}(171 x-116)+\left(\frac{8\left(117 x^{2}+118 x+81\right)}{9(x+1)}+\frac{128\left(x^{2}+1\right) H_{-1}}{3(x+1)}\right.\right. \\
& \left.+\frac{16(x+3)(3 x+1) H_{0}}{3(x+1)}+\frac{32}{3}(x+1) H_{1}\right) \zeta_{2}-\frac{4}{3}(107 x+89) H_{0} \\
& +\frac{256\left(4 x^{2}+3 x+4\right) H_{-1} H_{0}}{9(x+1)}-\frac{4\left(201 x^{2}+250 x+129\right) H_{0}^{2}}{9(x+1)}
\end{aligned}
$$




$$
\begin{aligned}
& +\frac{32\left(x^{2}+1\right) H_{-1} H_{0}^{2}}{3(x+1)}-\frac{32\left(3 x^{2}+4 x+3\right) H_{0}^{3}}{9(x+1)}-\frac{4}{9}(327 x-73) H_{1} \\
& -\frac{32}{9}(38 x+17) H_{0} H_{1}-\frac{80}{3}(x+1) H_{0}^{2} H_{1}-\frac{16}{3}(7 x+3) H_{1}^{2}-\frac{16}{3}(x+1) H_{0} H_{1}^{2} \\
& -\frac{256\left(4 x^{2}+3 x+4\right) H_{0,-1}}{9(x+1)}+\frac{280}{9}(x+1) H_{0,1}-\frac{128\left(x^{2}+1\right) H_{-1} H_{0,1}}{3(x+1)} \\
& +\frac{32}{3}(x+1) H_{0} H_{0,1}+\frac{128\left(x^{2}+1\right) H_{0,-1,1}}{3(x+1)}-\frac{64\left(x^{2}+1\right) H_{0,0,-1}}{3(x+1)} \\
& \left.+\frac{16\left(3 x^{2}-2 x+3\right) H_{0,0,1}}{3(x+1)}+\frac{128\left(x^{2}+1\right) H_{0,1,-1}}{3(x+1)}+\frac{16\left(x^{2}+14 x+1\right) \zeta_{3}}{3(x+1)}\right) \\
& +L_{Q}\left(-\frac{8}{27}(1925 x-284)-\frac{64}{9} \frac{\left(36 x^{3}+61 x^{2}+18 x+13\right) H_{-1} H_{0}}{x+1}\right. \\
& +\frac{64}{9} \frac{\left(36 x^{3}+61 x^{2}+18 x+13\right) H_{0,-1}}{x+1}+\frac{4}{9} \frac{\left(288 x^{3}+801 x^{2}+742 x+309\right) H_{0}^{2}}{x+1} \\
& +\left(-\frac{32}{9} \frac{\left(72 x^{3}+199 x^{2}+180 x+73\right)}{x+1}+\frac{64\left(7 x^{2}+6 x+3\right) H_{-1}}{3(x+1)}\right. \\
& \left.-\frac{16\left(35 x^{2}+66 x+35\right) H_{0}}{3(x+1)}+32(x-3) H_{1}\right) \zeta_{2}+\frac{8\left(186 x^{2}+211 x+73\right) H_{0}}{9(x+1)} \\
& +\frac{64\left(7 x^{2}+6 x+3\right) H_{-1}^{2} H_{0}}{3(x+1)}-\frac{32\left(11 x^{2}+6 x+7\right) H_{-1} H_{0}^{2}}{3(x+1)}+\frac{8}{27}(425 x+434) H_{1} \\
& +\frac{32\left(9 x^{2}+13 x+9\right) H_{0}^{3}}{9(x+1)}+\frac{16}{9}(193 x+121) H_{0} H_{1}+\frac{32}{3}(x+7) H_{0}^{2} H_{1} \\
& +\frac{32}{3}(15 x+8) H_{1}^{2}+64(x+1) H_{0} H_{1}^{2}+\frac{160}{9}(x+1) H_{1}^{3}+\frac{16}{9}(61 x+13) H_{0,1} \\
& -\frac{128\left(7 x^{2}+6 x+3\right) H_{-1} H_{0,-1}}{3(x+1)}-\frac{128 x(3 x+5) H_{0} H_{0,-1}}{3(x+1)}+\frac{16}{3}(25 x+1) H_{0} H_{0,1} \\
& -\frac{32}{3}(x+1) H_{1} H_{0,1}+\frac{128\left(7 x^{2}+6 x+3\right) H_{0,-1,-1}}{3(x+1)}+\frac{64\left(23 x^{2}+26 x+7\right) H_{0,0,-1}}{3(x+1)} \\
& \left.-\frac{16}{3}(19 x-5) H_{0,0,1}+48(x+1) H_{0,1,1}-\frac{32\left(21 x^{2}+38 x+25\right) \zeta_{3}}{3(x+1)}\right) \\
& +\left(\frac{4\left(1619 x^{2}+1338 x+1511\right)}{81(x+1)}+\frac{64\left(29 x^{2}+18 x+29\right) H_{-1}}{27(x+1)}+\frac{64\left(x^{2}+1\right) H_{-1}^{2}}{9(x+1)}\right. \\
& +\frac{4\left(147 x^{2}+298 x-9\right) H_{0}}{27(x+1)}+\frac{64\left(x^{2}+1\right) H_{-1} H_{0}}{9(x+1)}+\frac{4(x+5)(5 x+1) H_{0}^{2}}{9(x+1)} \\
& \left.+\frac{16}{9}(x+9) H_{1}-\frac{16}{3}(x+1) H_{0} H_{1}-\frac{64}{9}(x+1) H_{1}^{2}+\frac{16}{9}(x+1) H_{0,1}\right) \zeta_{2} \\
& +\frac{1}{x+1} \frac{8}{45}\left(131 x^{2}+178 x+131\right) \zeta_{2}^{2}-\left(\frac{8\left(235 x^{2}+404 x+409\right)}{27(x+1)}+\frac{80}{9}(x+1) H_{1}\right. \\
& \left.+\frac{256\left(x^{2}+1\right) H_{-1}}{9(x+1)}-\frac{8\left(15 x^{2}+22 x+15\right) H_{0}}{9(x+1)}\right) \zeta_{3}-\frac{32}{3} B_{4}(x+1) \\
& +\frac{11}{27}(1093 x-378)-\frac{(10159 x+8999)}{81} H_{0}+\frac{64\left(199 x^{2}+174 x+199\right) H_{-1} H_{0}}{81(x+1)}
\end{aligned}
$$




$$
\begin{aligned}
& -\frac{64}{9}(x+1) H_{-1}^{2} H_{0}+\frac{128\left(x^{2}+1\right) H_{-1}^{3} H_{0}}{27(x+1)}-\frac{2\left(4107 x^{2}+5327 x+3012\right) H_{0}^{2}}{81(x+1)} \\
& +\frac{32\left(19 x^{2}+18 x+19\right) H_{-1} H_{0}^{2}}{27(x+1)}-\frac{32\left(x^{2}+1\right) H_{-1}^{2} H_{0}^{2}}{9(x+1)}+\frac{1}{27}(1503-3905 x) H_{1} \\
& -\frac{2\left(903 x^{2}+1126 x+543\right) H_{0}^{3}}{81(x+1)}+\frac{64\left(x^{2}+1\right) H_{-1} H_{0}^{3}}{27(x+1)}-\frac{\left(51 x^{2}+70 x+51\right) H_{0}^{4}}{27(x+1)} \\
& -\frac{32}{81}(319 x+190) H_{0} H_{1}-\frac{40}{27}(31 x+16) H_{0}^{2} H_{1}-\frac{152}{27}(x+1) H_{0}^{3} H_{1} \\
& -\frac{8}{9}(3 x+55) H_{1}^{2}-\frac{8}{9}(9 x+1) H_{0} H_{1}^{2}+\frac{4}{9}(x+1) H_{0}^{2} H_{1}^{2}-\frac{64}{27}(x+1) H_{0} H_{1}^{3} \\
& -\frac{64\left(199 x^{2}+174 x+199\right) H_{0,-1}}{81(x+1)}+\frac{128}{9}(x+1) H_{-1} H_{0,-1}+\frac{4}{27}(311 x+467) H_{0,1} \\
& -\frac{128\left(x^{2}+1\right) H_{-1}^{2} H_{0,-1}}{9(x+1)}-\frac{512\left(4 x^{2}+3 x+4\right) H_{-1} H_{0,1}}{27(x+1)}+\frac{16}{27}(19 x+52) H_{0} H_{0,1} \\
& +\frac{16}{3}(x+1) H_{0}^{2} H_{0,1}+\frac{64}{9}(x-1) H_{1} H_{0,1}-\frac{80}{9}(x+1) H_{0} H_{1} H_{0,1} \\
& +\frac{64}{9}(x+1) H_{1}^{2} H_{0,1}+\frac{8}{9}(x+1) H_{0,1}^{2}-\frac{128}{9}(x+1) H_{0,-1,-1} \\
& +\frac{256\left(x^{2}+1\right) H_{-1} H_{0,-1,-1}}{9(x+1)}+\frac{512\left(4 x^{2}+3 x+4\right) H_{0,-1,1}}{27(x+1)} \\
& -\frac{64\left(19 x^{2}+18 x+19\right) H_{0,0,-1}}{27(x+1)}+\frac{128\left(x^{2}+1\right) H_{-1} H_{0,0,-1}}{9(x+1)}-\frac{64}{9}(x+1) H_{0} H_{0,0,1} \\
& +\frac{4\left(321 x^{2}+58 x+57\right) H_{0,0,1}}{27(x+1)}-\frac{128\left(x^{2}+1\right) H_{-1} H_{0,0,1}}{9(x+1)}+\frac{128}{9}(x+1) H_{1} H_{0,0,1} \\
& +\frac{512\left(4 x^{2}+3 x+4\right) H_{0,1,-1}}{27(x+1)}-\frac{32}{9}(13 x+1) H_{0,1,1}+\frac{256\left(x^{2}+1\right) H_{-1} H_{0,1,1}}{9(x+1)} \\
& +\frac{16}{3}(x+1) H_{0} H_{0,1,1}-\frac{32}{3}(x+1) H_{1} H_{0,1,1}-\frac{256\left(x^{2}+1\right) H_{0,-1,-1,-1}}{9(x+1)} \\
& -\frac{256\left(x^{2}+1\right) H_{0,-1,1,1}}{9(x+1)}-\frac{128\left(x^{2}+1\right) H_{0,0,-1,-1}}{9(x+1)}+\frac{128\left(x^{2}+1\right) H_{0,0,-1,1}}{9(x+1)} \\
& -\frac{128\left(x^{2}+1\right) H_{0,0,0,-1}}{9(x+1)}+\frac{8\left(21 x^{2}+10 x+21\right) H_{0,0,0,1}}{9(x+1)}+\frac{128\left(x^{2}+1\right) H_{0,0,1,-1}}{9(x+1)} \\
& \left.-\frac{32\left(7 x^{2}+6 x+7\right) H_{0,0,1,1}}{9(x+1)}-\frac{256\left(x^{2}+1\right) H_{0,1,-1,1}}{9(x+1)}-\frac{256\left(x^{2}+1\right) H_{0,1,1,-1}}{9(x+1)}\right] \\
& +C_{A} C_{F} T_{F}\left[\frac{88}{27} L_{M}^{3}(x+1)+\frac{176}{27} L_{Q}^{3}(x+1)+L_{M}^{2}\left(\frac{32}{3}(x+1) H_{0}-\frac{4(83 x-37)}{9}\right.\right. \\
& \left.-\frac{32\left(x^{2}+1\right) H_{-1} H_{0}}{3(x+1)}-\frac{16 x H_{0}^{2}}{3(x+1)}+\frac{32\left(x^{2}+1\right) H_{0,-1}}{3(x+1)}+\frac{32 x \zeta_{2}}{3(x+1)}\right) \\
& -L_{Q}^{2}\left(\frac{4}{27}(997 x+241)+\frac{256}{9}(x+1) H_{0}+\frac{32\left(x^{2}+1\right) H_{-1} H_{0}}{3(x+1)}+\frac{16 x H_{0}^{2}}{3(x+1)}\right. \\
& \left.+\frac{176}{9}(x+1) H_{1}-\frac{32\left(x^{2}+1\right) H_{0,-1}}{3(x+1)}-\frac{32 x \zeta_{2}}{3(x+1)}\right)+L_{M}\left(-\frac{4}{81}(4577 x-4267)\right.
\end{aligned}
$$




$$
\begin{aligned}
& +\left(\frac{16\left(3 x^{2}+14 x-9\right)}{9(x+1)}-\frac{64\left(x^{2}+1\right) H_{-1}}{3(x+1)}+\frac{16\left(3 x^{2}+4 x+3\right) H_{0}}{3(x+1)}\right) \zeta_{2} \\
& -\frac{16}{27}(29 x-109) H_{0}-\frac{128\left(4 x^{2}+3 x+4\right) H_{-1} H_{0}}{9(x+1)}+\frac{4\left(19 x^{2}+4 x+25\right) H_{0}^{2}}{9(x+1)} \\
& -\frac{16\left(x^{2}+1\right) H_{-1} H_{0}^{2}}{3(x+1)}-\frac{32 x H_{0}^{3}}{9(x+1)}+\frac{32}{3}(x-1) H_{1}+8(x+1) H_{0}^{2} H_{1} \\
& +\frac{128\left(4 x^{2}+3 x+4\right) H_{0,-1}}{9(x+1)}-\frac{16}{3}(x+1) H_{0,1}+\frac{64\left(x^{2}+1\right) H_{-1} H_{0,1}}{3(x+1)} \\
& -16(x+1) H_{0} H_{0,1}-\frac{64\left(x^{2}+1\right) H_{0,-1,1}}{3(x+1)}+\frac{32\left(x^{2}+1\right) H_{0,0,-1}}{3(x+1)}+\frac{64 x H_{0,0,1}}{3(x+1)} \\
& \left.-\frac{64\left(x^{2}+1\right) H_{0,1,-1}}{3(x+1)}-\frac{32\left(x^{2}+3 x+1\right) \zeta_{3}}{x+1}\right)+L_{Q}\left(\frac{4}{81}(19751 x-2371)\right. \\
& +\frac{32}{9} \frac{\left(36 x^{3}+61 x^{2}+18 x+13\right) H_{-1} H_{0}}{x+1}-\frac{32}{9} \frac{\left(36 x^{3}+61 x^{2}+18 x+13\right) H_{0,-1}}{x+1} \\
& -\frac{8}{9} \frac{\left(72 x^{3}+39 x^{2}-89 x-36\right) H_{0}^{2}}{x+1}+\left(\frac{16}{9} \frac{\left(72 x^{3}+37 x^{2}-84 x-29\right)}{x+1}\right. \\
& \left.-\frac{32\left(7 x^{2}+6 x+3\right) H_{-1}}{3(x+1)}-\frac{16\left(3 x^{2}+8 x+3\right) H_{0}}{3(x+1)}-\frac{64}{3}(4 x+1) H_{1}\right) \zeta_{2} \\
& +\frac{32\left(401 x^{2}+556 x+137\right) H_{0}}{27(x+1)}+\frac{80 x H_{0}^{3}}{9(x+1)}-\frac{32\left(7 x^{2}+6 x+3\right) H_{-1}^{2} H_{0}}{3(x+1)} \\
& +\frac{16\left(11 x^{2}+6 x+7\right) H_{-1} H_{0}^{2}}{3(x+1)}+\frac{8}{27}(1195 x+169) H_{1}+\frac{352}{9}(x+1) H_{0} H_{1} \\
& +\frac{8}{3}(11 x-1) H_{0}^{2} H_{1}+\frac{176}{9}(x+1) H_{1}^{2}-\frac{16}{3}(x+1) H_{0} H_{1}^{2}+\frac{208}{9}(x+1) H_{0,1} \\
& +\frac{64\left(7 x^{2}+6 x+3\right) H_{-1} H_{0,-1}}{3(x+1)}+\frac{64 x(3 x+5) H_{0} H_{0,-1}}{3(x+1)}-\frac{16}{3}(11 x-1) H_{0} H_{0,1} \\
& +\frac{64}{3}(x+1) H_{1} H_{0,1}-\frac{64\left(7 x^{2}+6 x+3\right) H_{0,-1,-1}}{3(x+1)}-\frac{32\left(23 x^{2}+26 x+7\right) H_{0,0,-1}}{3(x+1)} \\
& \left.+\frac{32}{3}(7 x+1) H_{0,0,1}-32(x+1) H_{0,1,1}+\frac{64\left(3 x^{2}+5 x+4\right) \zeta_{3}}{3(x+1)}\right) \\
& +\left(\frac{16\left(174 x^{2}+209 x-189\right)}{81(x+1)}-\frac{32\left(29 x^{2}+18 x+29\right) H_{-1}}{27(x+1)}-\frac{32\left(x^{2}+1\right) H_{-1}^{2}}{9(x+1)}\right. \\
& +\frac{8\left(63 x^{2}+29 x+6\right) H_{0}}{27(x+1)}-\frac{32\left(x^{2}+1\right) H_{-1} H_{0}}{9(x+1)}+\frac{8\left(3 x^{2}+8 x+9\right) H_{0}^{2}}{9(x+1)} \\
& \left.-\frac{8}{9}(3 x+14) H_{1}+\frac{80}{9}(x+1) H_{0} H_{1}+\frac{64}{9}(x+1) H_{1}^{2}-\frac{16}{9}(7 x+1) H_{0,1}\right) \zeta_{2} \\
& -\frac{16}{15(x+1)}\left(36 x^{2}+51 x+22\right) \zeta_{2}^{2}+\left(\frac{2\left(497 x^{2}+1102 x+1085\right)}{27(x+1)}+\frac{16}{9}(x+1) H_{1}\right. \\
& \left.+\frac{128\left(x^{2}+1\right) H_{-1}}{9(x+1)}+\frac{32\left(6 x^{2}+4 x-3\right) H_{0}}{9(x+1)}\right) \zeta_{3}+\frac{16}{3} B_{4}(x+1) \\
& -\frac{4}{81}(995 x-2807) H_{0}-\frac{32\left(199 x^{2}+174 x+199\right) H_{-1} H_{0}}{81(x+1)}+\frac{32}{9}(x+1) H_{-1}^{2} H_{0}
\end{aligned}
$$




$$
\begin{aligned}
& -\frac{64\left(x^{2}+1\right) H_{-1}^{3} H_{0}}{27(x+1)}+\frac{4\left(253 x^{2}+391 x+586\right) H_{0}^{2}}{81(x+1)}+\frac{16\left(x^{2}+1\right) H_{-1}^{2} H_{0}^{2}}{9(x+1)} \\
& -\frac{16\left(19 x^{2}+18 x+19\right) H_{-1} H_{0}^{2}}{27(x+1)}+\frac{8\left(22 x^{2}+7 x+25\right) H_{0}^{3}}{81(x+1)}-\frac{32\left(x^{2}+1\right) H_{-1} H_{0}^{3}}{27(x+1)} \\
& -\frac{16 x H_{0}^{4}}{27(x+1)}-\frac{8}{27}(65 x-29) H_{1}+\frac{8}{9}(9 x+4) H_{0} H_{1}+\frac{8}{9}(14 x+3) H_{0}^{2} H_{1} \\
& +\frac{56}{27}(x+1) H_{0}^{3} H_{1}-\frac{4}{9}(43 x-46) H_{1}^{2}-\frac{8}{9}(2 x+5) H_{0} H_{1}^{2}-\frac{4}{9}(x+1) H_{0}^{2} H_{1}^{2} \\
& +\frac{32}{27}(x+1) H_{0} H_{1}^{3}+\frac{32\left(199 x^{2}+174 x+199\right) H_{0,-1}}{81(x+1)}-\frac{64}{9}(x+1) H_{-1} H_{0,-1} \\
& +\frac{64\left(x^{2}+1\right) H_{-1}^{2} H_{0,-1}}{9(x+1)}-\frac{8}{27}(143 x+2) H_{0,1}+\frac{256\left(4 x^{2}+3 x+4\right) H_{-1} H_{0,1}}{27(x+1)} \\
& -\frac{16}{9}(13 x+6) H_{0} H_{0,1}-\frac{8}{3}(x+1) H_{0}^{2} H_{0,1}+\frac{8}{9}(11 x+20) H_{1} H_{0,1} \\
& +\frac{64}{9}(x+1) H_{0} H_{1} H_{0,1}-\frac{64}{9}(x+1) H_{1}^{2} H_{0,1}+\frac{16}{9}(7 x+1) H_{0,1}^{2} \\
& +\frac{64}{9}(x+1) H_{0,-1,-1}-\frac{128\left(x^{2}+1\right) H_{-1} H_{0,-1,-1}}{9(x+1)}-\frac{256\left(4 x^{2}+3 x+4\right) H_{0,-1,1}}{27(x+1)} \\
& +\frac{32\left(19 x^{2}+18 x+19\right) H_{0,0,-1}}{27(x+1)}-\frac{64\left(x^{2}+1\right) H_{-1} H_{0,0,-1}}{9(x+1)} \\
& +\frac{8\left(9 x^{2}+101 x+12\right) H_{0,0,1}}{27(x+1)}+\frac{64\left(x^{2}+1\right) H_{-1} H_{0,0,1}}{9(x+1)}-\frac{16}{9}(7 x+1) H_{0} H_{0,0,1} \\
& -\frac{160}{9}(x+1) H_{1} H_{0,0,1}-\frac{256\left(4 x^{2}+3 x+4\right) H_{0,1,-1}}{27(x+1)}-\frac{16}{9}(x+7) H_{0,1,1} \\
& -\frac{128\left(x^{2}+1\right) H_{-1} H_{0,1,1}}{9(x+1)}-\frac{16}{9}(11 x+5) H_{0} H_{0,1,1}+16(x+1) H_{1} H_{0,1,1} \\
& +\frac{128\left(x^{2}+1\right) H_{0,-1,-1,-1}}{9(x+1)}+\frac{128\left(x^{2}+1\right) H_{0,-1,1,1}}{9(x+1)}+\frac{64\left(x^{2}+1\right) H_{0,0,-1,-1}}{9(x+1)} \\
& -\frac{64\left(x^{2}+1\right) H_{0,0,-1,1}}{9(x+1)}+\frac{64\left(x^{2}+1\right) H_{0,0,0,-1}}{9(x+1)}+\frac{64\left(5 x^{2}+6 x-1\right) H_{0,0,0,1}}{9(x+1)} \\
& -\frac{64\left(x^{2}+1\right) H_{0,0,1,-1}}{9(x+1)}-\frac{16\left(x^{2}-2 x-11\right) H_{0,0,1,1}}{9(x+1)}+\frac{128\left(x^{2}+1\right) H_{0,1,-1,1}}{9(x+1)} \\
& \left.+\frac{128\left(x^{2}+1\right) H_{0,1,1,-1}}{9(x+1)}-\frac{112}{9}(x+1) H_{0,1,1,1}-\frac{2}{729}(108295 x-86681)\right] \\
& +C_{F} T_{F}^{2}\left[-\frac{64}{27} L_{M}^{3}(x+1)-\frac{32}{27} L_{Q}^{3}(x+1)-L_{M}^{2}\left(\frac{32}{27}(11 x-1)\right.\right. \\
& \left.+\frac{32}{9}(x+1) H_{0}\right)+L_{Q}^{2}\left(\frac{32}{27}(17 x+8)+\frac{64}{9}(x+1) H_{0}+\frac{32}{9}(x+1) H_{1}\right) \\
& -\frac{992}{81} L_{M}(x+1)+L_{Q}\left(-\frac{32}{81}(280 x+37)-\frac{64}{27}(34 x+19) H_{0}-\frac{32}{3}(x+1) H_{0}^{2}\right. \\
& -\frac{64}{27}(17 x+8) H_{1}-\frac{64}{9}(x+1) H_{0} H_{1}-\frac{32}{9}(x+1) H_{1}^{2}-\frac{64}{9}(x+1) H_{0,1}
\end{aligned}
$$




$$
\begin{aligned}
& \left.+\frac{128}{9}(x+1) \zeta_{2}\right)+\frac{64}{81}(6 x-7) H_{0}+\frac{16}{81}(11 x-1) H_{0}^{2}+\frac{16}{81}(x+1) H_{0}^{3} \\
& \left.-\frac{448}{27}(x+1) \zeta_{3}+\frac{16}{729}(431 x+323)\right] \\
& +C_{F} N_{F} T_{F}^{2}\left[-\frac{32}{27} L_{M}^{3}(x+1)-\frac{64}{27} L_{Q}^{3}(x+1)+L_{Q}^{2}\left(\frac{64}{27}(17 x+8)\right.\right. \\
& \left.+\frac{128}{9}(x+1) H_{0}+\frac{64}{9}(x+1) H_{1}\right)+L_{M}\left(\frac{32}{81}(5 x-73)+\frac{32}{27}(11 x-1) H_{0}\right. \\
& \left.+\frac{16}{9}(x+1) H_{0}^{2}\right)+L_{Q}\left(-\frac{64}{81}(280 x+37)-\frac{128}{27}(34 x+19) H_{0}-\frac{64}{3}(x+1) H_{0}^{2}\right. \\
& -\frac{128}{27}(17 x+8) H_{1}-\frac{128}{9}(x+1) H_{0} H_{1}-\frac{64}{9}(x+1) H_{1}^{2}-\frac{128}{9}(x+1) H_{0,1} \\
& \left.+\frac{256}{9}(x+1) \zeta_{2}\right)+\frac{128}{81}(6 x-7) H_{0}+\frac{32}{81}(11 x-1) H_{0}^{2}+\frac{32}{81}(x+1) H_{0}^{3} \\
& \left.\left.+\frac{256}{27}(x+1) \zeta_{3}-\frac{64}{729}(161 x+215)\right]\right\}+\hat{c}_{q, 2}^{(3)} .
\end{aligned}
$$

Here the +-prescription is defined by

$$
\int_{0}^{1} d x g(x)[f(x)]_{+}=\int_{0}^{1} d x[g(x)-g(1)] f(x) .
$$

The contribution of the massive Wilson coefficient $H_{q, 2}$ is found by combining the massless Wilson coefficient $C_{q, 2}$ and $L_{q, 2}$ by

$$
H_{q, 2}\left(x, Q^{2}\right)=C_{q, 2}\left(N_{F}, x, Q^{2}\right)+L_{q, 2}\left(x, Q^{2}\right) .
$$

Except for $c_{q, 2}^{(3)}\left(N_{F}\right)$, the Wilson coefficients are expressed by up to weight $\mathrm{w}=4$ harmonic polylogarithms. Note the emergence of a denominator $1 /(1-x)^{2}$, cf. [19], which is properly regularized by its numerator function in the limit $x \rightarrow 1$. We note that we have applied the shuffle algebra, cf. [28], which leads to a reduction of the number of harmonic polylogarithms compared to the linear representation, making the numerical evaluation faster.

\section{Numerical Results}

In the following we illustrate the asymptotic charm corrections up to 3-loop order to the charged current non-singlet combinations $F_{1,2}^{W^{+}-W^{-}}\left(x, Q^{2}\right)$ choosing the renormalization and factorization scales $\mu^{2}=Q^{2}$. First we consider the behaviour of the corrections at small and large values of the Bjorken variable $x$. For those of the massless 3-loop Wilson coefficients see [3]. The limiting behaviour for the two contributing functions $L_{q, i}^{W^{+}-W^{-}, \mathrm{NS}}\left(N_{F}+1\right)-\hat{C}_{q, i}^{W^{+}-W^{-}, \mathrm{NS}}\left(N_{F}\right)$ and $H_{q, i}^{W^{+}-W^{-}, \mathrm{NS}}\left(N_{F}+1\right)-C_{q, i}^{W^{+}-W^{-}, \mathrm{NS}}\left(N_{F}+1\right)$ are the same, see also 15 .

For the 3-loop contributions, yet for general values of $\mu^{2}$, at low values of $x$ one has

$$
\begin{aligned}
L_{q, L}^{W^{+}-W^{-} \mathrm{NS}}\left(N_{F}+1\right)-\hat{C}_{q, L}^{W^{+}-W^{-}, \mathrm{NS}}\left(N_{F}\right) & \propto a_{s}^{3} \frac{8}{3} C_{F}^{2} T_{F} \ln ^{2}(x) \\
L_{q, 2}^{W^{+}-W^{-}, \mathrm{NS}}\left(N_{F}+1\right)-\hat{C}_{q, 2}^{W^{+}-W^{-}, \mathrm{NS}}\left(N_{F}\right) & \propto a_{s}^{3}\left\{\frac{16}{27} C_{A} C_{F} T_{F}-\frac{5}{9} C_{F}^{2} T_{F}\right\} \ln ^{4}(x)
\end{aligned}
$$


and at large $x$

$$
\begin{aligned}
L_{q, L}^{W^{+}-W^{-}, \mathrm{NS}}\left(N_{F}+1\right)-\hat{C}_{q, L}^{W^{+}-W^{-}, \mathrm{NS}}\left(N_{F}\right) \propto & a_{s}^{3} C_{F} T_{F}\left\{\frac{128}{3} C_{F} L_{Q} \ln ^{2}(1-x)+\left[C _ { F } \left(\frac{896}{27}\right.\right.\right. \\
& \left.+\frac{320}{9} L_{M}+\frac{32}{3} L_{M}^{2}\right)+32 C_{F} L_{Q}^{2}+\left(-\frac{1088}{9} C_{A}\right. \\
& +\frac{80}{9} C_{F}+\frac{128}{9} T_{F}+\frac{256}{9} N_{F} T_{F}+\frac{128}{3} \zeta_{2} C_{A} \\
& \left.\left.\left.-\frac{256}{3} \zeta_{2} C_{F}\right) L_{Q}\right] \ln (1-x)\right\} \\
L_{q, 2}^{W^{+}-W^{-}, \mathrm{NS}}\left(N_{F}+1\right)-\hat{C}_{q, 2}^{W^{+}-W^{-}, \mathrm{NS}}\left(N_{F}\right) \propto & a_{s}^{3} C_{F} T_{F}\left\{\frac{320}{9} C_{F} L_{Q}\left(\frac{\ln ^{3}(1-x)}{1-x}\right)_{+}\right. \\
& +\left[C_{F}\left(\frac{448}{9}+\frac{160}{3} L_{M}+16 L_{M}^{2}\right)+48 C_{F} L_{Q}^{2}\right. \\
& +\left(-\frac{352}{9} C_{A}-\frac{512}{3} C_{F}+\frac{64}{9} T_{F}\right. \\
& \left.\left.\left.+\frac{128}{9} N_{F} T_{F}\right) L_{Q}\right]\left(\frac{\ln ^{2}(1-x)}{1-x}\right)\right\}
\end{aligned}
$$

Below we plot the heavy flavor contribution to the structure function $F_{1}^{W^{+}-W^{-}}\left(x, Q^{2}\right)$ for the quark mass $m_{c}=1.59 \mathrm{GeV}$ in the on-shell scheme [29] and the scales $Q^{2}=\mu^{2}=$ $10,100,1000 \mathrm{GeV}^{2}$ for the complete structure function, including the massive and massless terms.

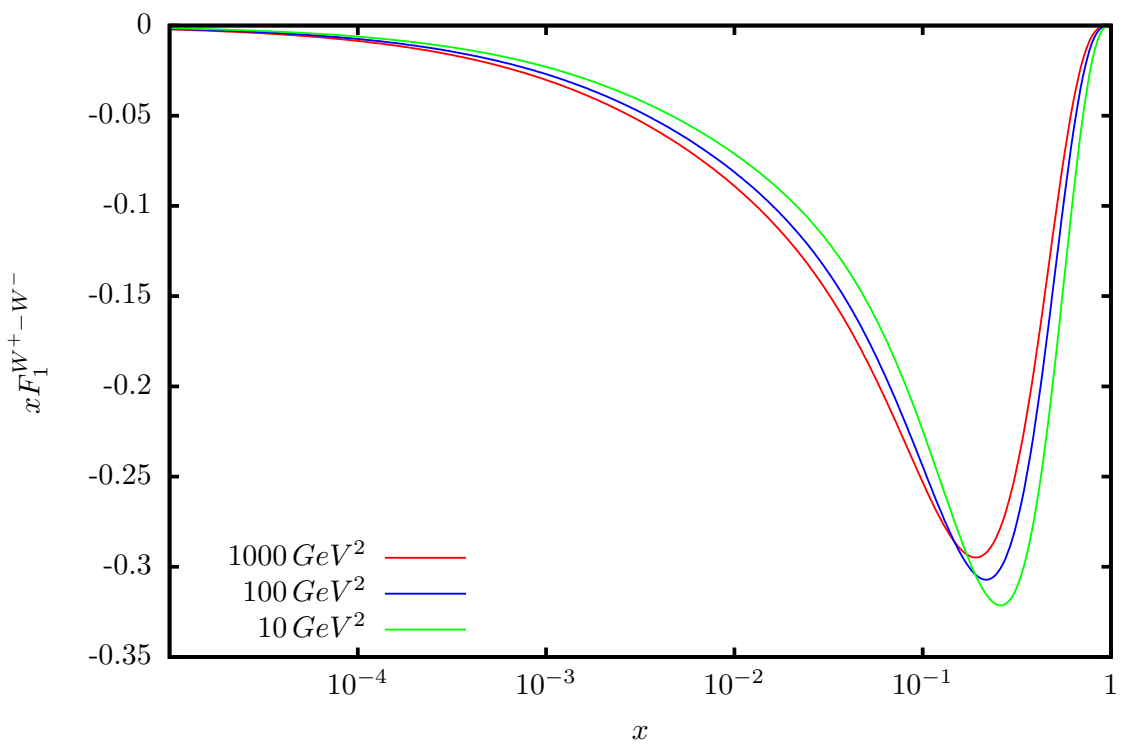

Figure 1: The structure function $x F_{1}^{W^{+}-W^{-}}\left(x, Q^{2}\right)$, containing the 3-loop corrections including the asymptotic corrections for charm using $m_{c}^{\mathrm{OMS}}=1.59 \mathrm{GeV}$ and the PDFs [30]. 


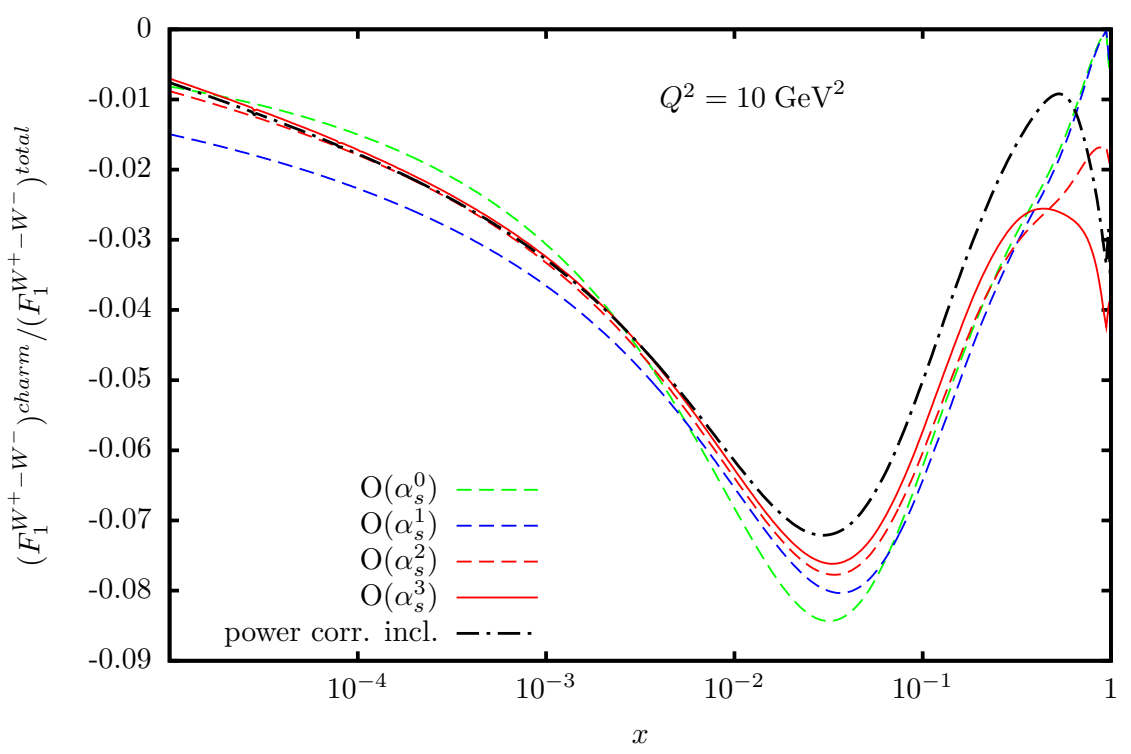

Figure 2: The ratio of massive contributions to the structure function $x F_{1}^{W^{+}-W^{-}}\left(x, Q^{2}\right)$ over the complete structure function for $Q^{2}=10 \mathrm{GeV}^{2}$, containing the 3-loop corrections including the asymptotic corrections for charm using $m_{c}^{\mathrm{OMS}}=1.59 \mathrm{GeV}$ and the PDFs [30]. For the dash-dotted line, asymptotic corrections at three loops and the complete heavy flavor contributions up to $O\left(a_{s}^{2}\right)$ [15] are taken into account.

In Figure 1 the scale evolution of the structure function $x F_{1}^{W^{+}-W^{-}}\left(x, Q^{2}\right)$ is shown in the range $Q^{2} \in[10,1000] \mathrm{GeV}^{2}$, including the asymptotic charm quark corrections to 3-loop order.

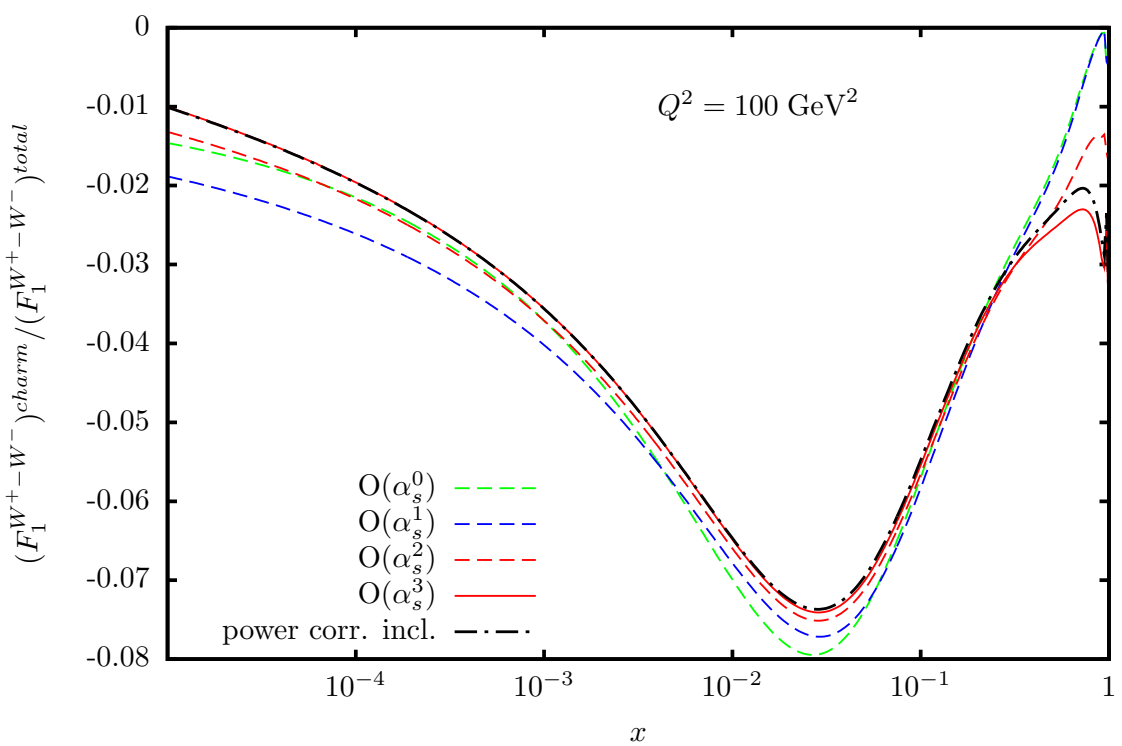

Figure 3: The ratio of massive contributions to the structure function $x F_{1}^{W^{+}-W^{-}}\left(x, Q^{2}\right)$ over the complete structure function for $Q^{2}=100 \mathrm{GeV}^{2}$, containing the 3-loop corrections including the asymptotic corrections for charm using $m_{c}^{\mathrm{OMS}}=1.59 \mathrm{GeV}$ and the PDFs [30]. For the dash-dotted line, asymptotic corrections at three loops and the complete heavy flavor contributions up to $O\left(a_{s}^{2}\right)[15$ are taken into account. 


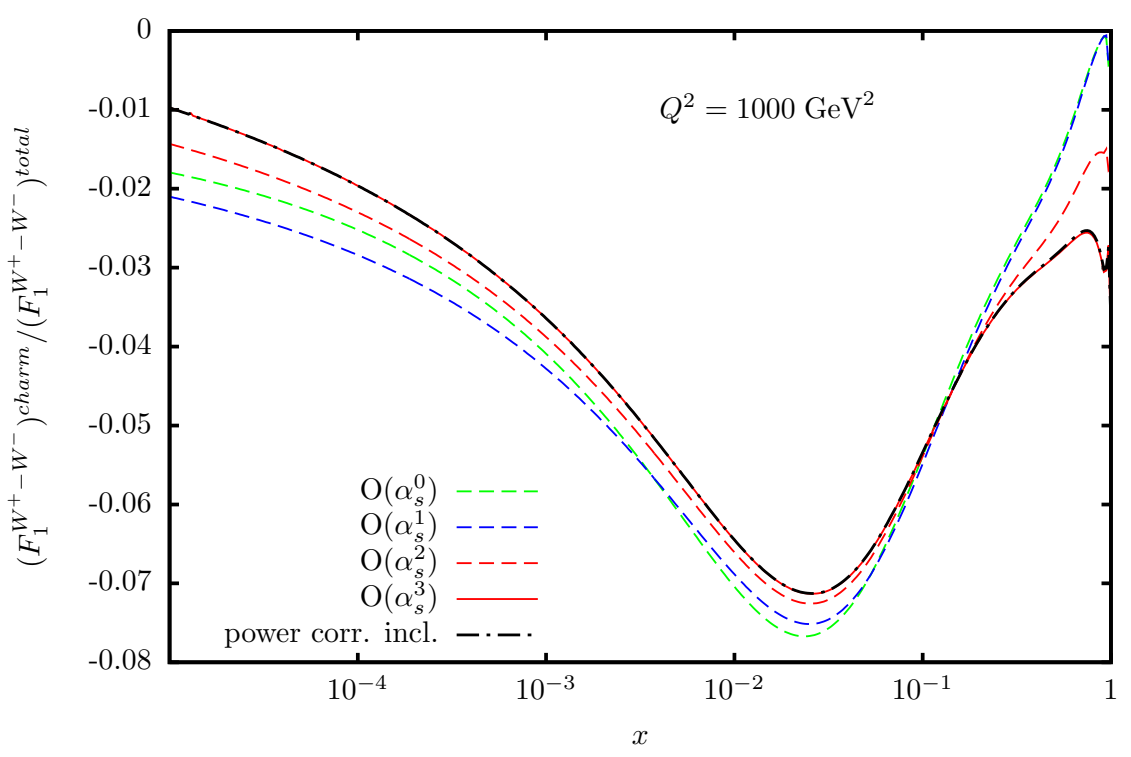

Figure 4: The ratio of massive contributions to the structure function $x F_{1}^{W^{+}-W^{-}}\left(x, Q^{2}\right)$ over the complete structure function for $Q^{2}=1000 \mathrm{GeV}^{2}$, containing the 3-loop corrections including the asymptotic corrections for charm using $m_{c}^{\mathrm{OMS}}=1.59 \mathrm{GeV}$ and the PDFs [30]. For the dash-dotted line, asymptotic corrections at three loops and the complete heavy flavor contributions up to $O\left(a_{s}^{2}\right)$ [15] are taken into account.

Here and in the following we refer to the parton distribution functions [30]. As typical for nonsinglet contributions, the profile is shifted from larger to smaller values of $x$ with growing values of $Q^{2}$. However, the effects are much smaller than in the singlet case. As it is well known, the validity of the asymptotic charm quark corrections in the case of $F_{L}\left(x, Q^{2}\right)$, and therefore for $F_{2}$ and in part for $x F_{1}$, is setting in at higher scales only due to the $F_{L}$ contribution, for details see [14]. We will discuss these aspects in the following figures for $x F_{1}$ and $F_{2}$.

In Figure 2 the corrections to $x F_{1}^{W^{+}-W^{-}}\left(x, Q^{2}\right)$ are illustrated for $Q^{2}=10 \mathrm{GeV}^{2}$ by adding the contributions from $O\left(a_{s}^{0}\right)$ to $O\left(a_{s}^{3}\right)$, showing an increasing degree of stabilization. We also present the exact heavy flavor corrections to $O\left(a_{s}^{2}\right)$ [15], showing deviations in the range $x \gtrsim 10^{-2}$, while below there is exact agreement. The latter effect is due to the sufficiently large $W^{2}=Q^{2}(1-x) / x$ values through which the heavy quarks are made effectively massless for this structure function even at this low scale of $Q^{2}$. The charm quark corrections for $x F_{1}^{W^{+}-W^{-}}\left(x, Q^{2}\right)$ vary in a range of $-8 \%$ to $\sim 0 \%$, depending on $x$, with a maximal relative contribution around $x \sim 3 \cdot 10^{-2}$.

Figure 3 shows that at $Q^{2}=100 \mathrm{GeV}^{2}$ the asymptotic corrections agree also in the case where we include the power corrections to larger values of $x \sim 0.3$ and for $Q^{2}=1000 \mathrm{GeV}^{2}$, Figure 4, the agreement is obtained in the whole $x$ range.

We turn now to the numerical illustration of the structure function $F_{2}^{W^{+}-W^{-}}\left(x, Q^{2}\right)$. In Figure 5 we show the scaling violations of $F_{2}^{W^{+}-W^{-}}\left(x, Q^{2}\right)$ in the region $Q^{2} \in[10,1000] \mathrm{GeV}^{2}$, shifting the profile to lower values of $x$ with growing virtualities $Q^{2}$. Figure 6 shows the contributions to $F_{2}^{W^{+}-W^{-}}\left(x, Q^{2}\right)$ at $Q^{2}=10 \mathrm{GeV}^{2}$ for growing order in the strong coupling constant stabilizing at 3-loop order, except of very large values of $x$. At $Q^{2}=10 \mathrm{GeV}^{2}$ comparing the results for $2 x F_{1}$ and $F_{2}$ the effect of $F_{L}\left(x, Q^{2}\right)$ is clearly visible. The asymptotic expression is not yet valid in the charged current case, as the complete $O\left(a_{s}^{2}\right)$ charm quark corrections show. 


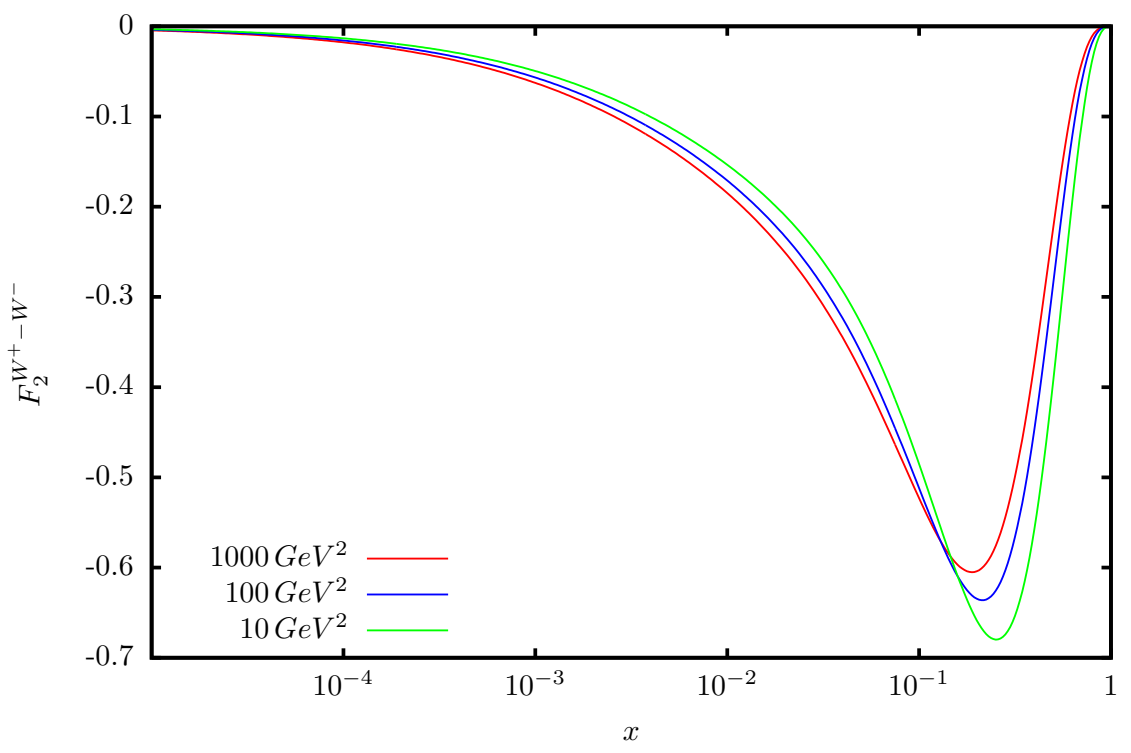

Figure 5: The structure function $F_{2}^{W^{+}-W^{-}}\left(x, Q^{2}\right)$, containing the 3-loop corrections including the asymptotic corrections for charm using $m_{c}^{\mathrm{OMS}}=1.59 \mathrm{GeV}$ and the PDFs [30].

Again the relative charm quark corrections vary in the range $[-8 \%, \sim 0 \%]$. As shown in Figure 7 , the asymptotic corrections agree with the case where the power corrections are included, except for a small range at very large $x$ values at $Q^{2}=100 \mathrm{GeV}^{2}$. Finally, this effect disappears for $Q^{2}=1000 \mathrm{GeV}^{2}$, see Figure 8 .

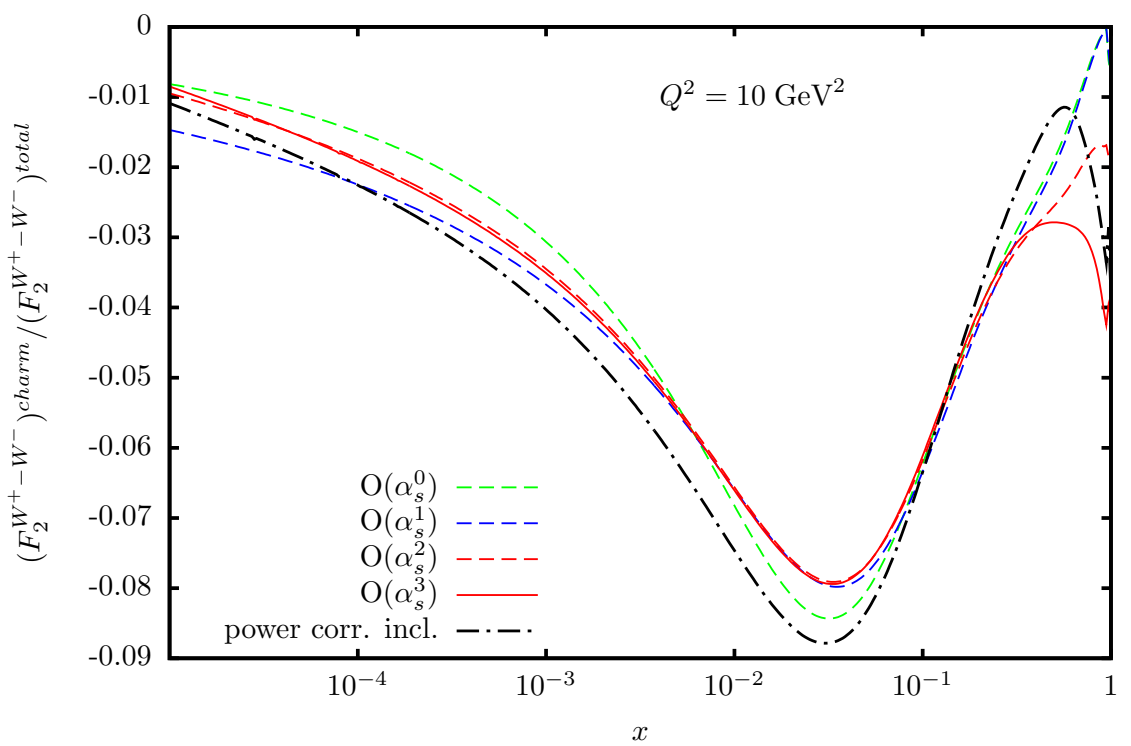

Figure 6: The ratio of massive contributions to the structure function $F_{2}^{W^{+}-W^{-}}\left(x, Q^{2}\right)$ over the complete structure function for $Q^{2}=10 \mathrm{GeV}^{2}$, containing the 3-loop corrections including the asymptotic corrections for charm using $m_{c}^{\mathrm{OMS}}=1.59 \mathrm{GeV}$ and the PDFs [30]. For the dash-dotted line, asymptotic corrections at three loops and the complete heavy flavor contributions up to $O\left(a_{s}^{2}\right)[15]$ are taken into account. 


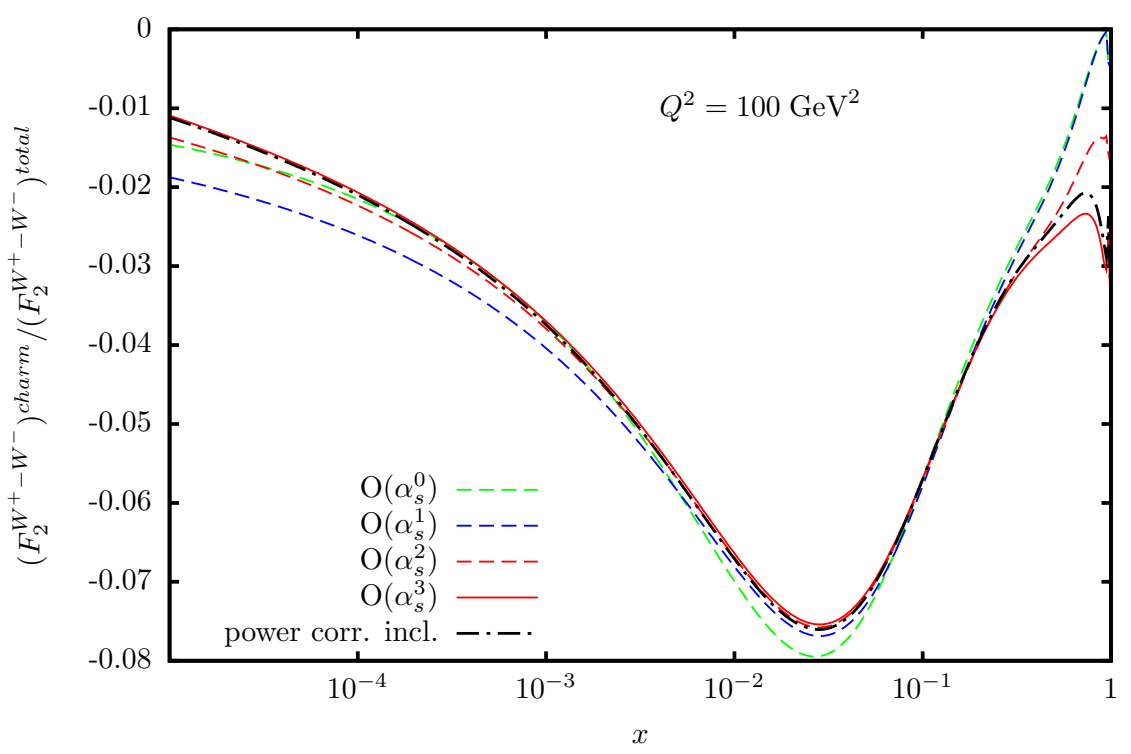

Figure 7: The ratio of massive contributions to the structure function $F_{2}^{W^{+}-W^{-}}\left(x, Q^{2}\right)$ over the complete structure function for $Q^{2}=100 \mathrm{GeV}^{2}$, containing the 3-loop corrections including the asymptotic corrections for charm using $m_{c}^{\mathrm{OMS}}=1.59 \mathrm{GeV}$ and the PDFs [30]. For the dash-dotted line, asymptotic corrections at three loops and the complete heavy flavor contributions up to $O\left(a_{s}^{2}\right)$ [15] are taken into account.

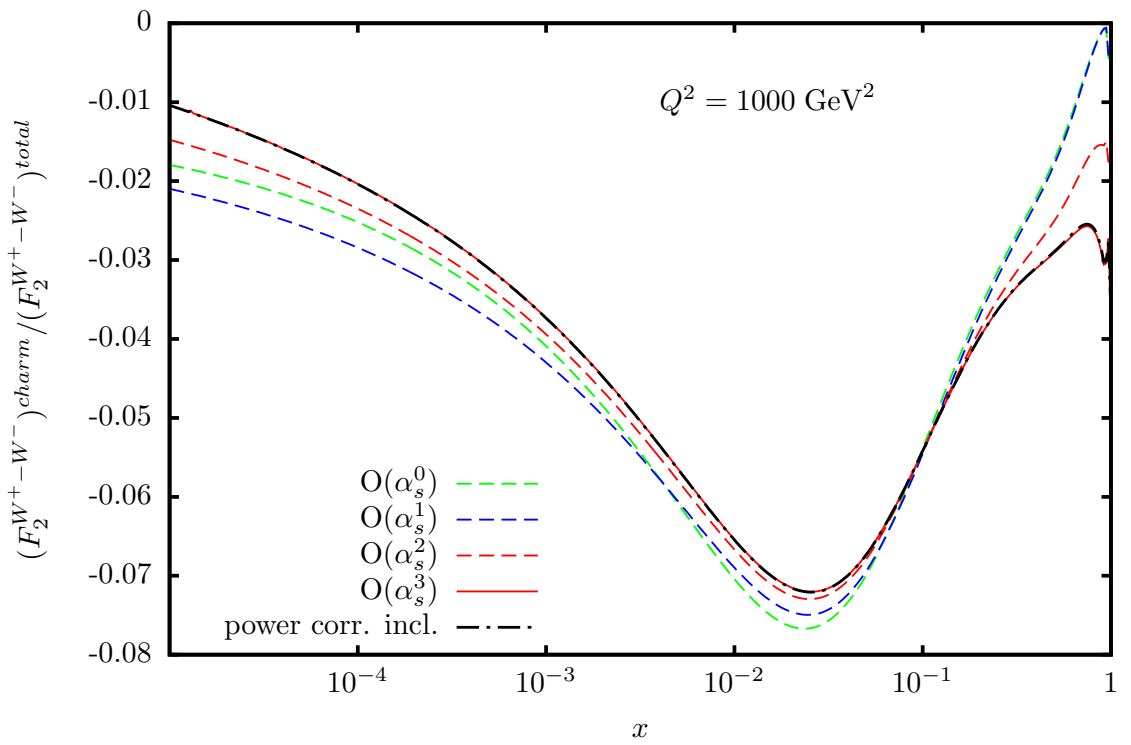

Figure 8: The ratio of massive contributions to the structure function $F_{2}^{W^{+}-W^{-}}\left(x, Q^{2}\right)$ over the complete structure function for $Q^{2}=1000 \mathrm{GeV}^{2}$, containing the 3-loop corrections including the asymptotic corrections for charm using $m_{c}^{\mathrm{OMS}}=1.59 \mathrm{GeV}$ and the PDFs [30]. For the dash-dotted line, asymptotic corrections at three loops and the complete heavy flavor contributions up to $O\left(a_{s}^{2}\right)$ [15] are taken into account. 


\section{The Sum Rules}

For the combination of the charged current structure functions being considered here, there exist sum rules arising from the lowest Mellin moment. In the case of $F_{2}^{W^{+}-W^{-}}\left(x, Q^{2}\right)$, one obtains the Adler sum rule [2] and for $F_{1}^{W^{+}-W^{-}}\left(x, Q^{2}\right)$ the unpolarized Bjorken sum rule [1], for which also the target mass corrections have to be considered, cf. [15.

The Adler sum rule states

$$
\int_{0}^{1} \frac{d x}{x}\left[F_{2}^{\bar{\nu} p}\left(x, Q^{2}\right)-F_{2}^{\nu p}\left(x, Q^{2}\right)\right]=2\left[1+\sin ^{2}\left(\theta_{c}\right)\right]
$$

for three massless flavors. Here $\theta_{c}$ denotes the Cabibbo angle [20]. The integral (5.1) neither receives QCD nor quark- or target mass corrections [17], cf. also [31,32]. Up to 2-loop order the vanishing of the heavy quark corrections has been shown in Ref. [15]. Considering the limit of large scales $Q^{2} \gg m^{2}$, this is confirmed at 3-loop order since the flavor non-singlet OMEs vanish for $N=1$ due to fermion number conservation [19] and the first moment of the corresponding massless Wilson coefficient also vanishes [33].

The unpolarized Bjorken sum rule [1] is given by

$$
\int_{0}^{1} d x\left[F_{1}^{\bar{\nu} p}\left(x, Q^{2}\right)-F_{1}^{\nu p}\left(x, Q^{2}\right)\right]=C_{\mathrm{uBJ}}\left(\hat{a}_{s}\right),
$$

with $\hat{a}_{s}=\alpha_{s} / \pi$. The massless 1-loop 4, 5, 34, 35, 2-loop [36], 3-loop 37] and 4-loop [38] QCD corrections have been calculated

$$
\begin{aligned}
C_{\mathrm{uBJ}}\left(\hat{a}_{s}\right),= & 1-0.66667 \hat{a}_{s}+\hat{a}_{s}^{2}\left(-3.83333+0.29630 N_{F}\right) \\
& +\hat{a}_{s}^{3}\left(-36.1549+6.33125 N_{F}-0.15947 N_{F}^{2}\right) \\
& +\hat{a}_{s}^{4}\left(-436.768+111.873 N_{F}-7.11450 N_{F}^{2}+0.10174 N_{F}^{3}\right),
\end{aligned}
$$

setting $\mu^{2}=Q^{2}$ for $S U(3)_{c}$. For $N_{F}=3,4$ the massless QCD corrections are given by

$$
\begin{aligned}
& C_{\mathrm{uBJ}}\left(\hat{a}_{s}, N_{F}=3\right)=1-0.66667 \hat{a}_{s}-2.94444 \hat{a}_{s}^{2}-18.5963 \hat{a}_{s}^{3}-162.436 \hat{a}_{s}^{4} \\
& C_{\mathrm{uBJ}}\left(\hat{a}_{s}, N_{F}=4\right)=1-0.66667 \hat{a}_{s}-2.64815 \hat{a}_{s}^{2}-13.3813 \hat{a}_{s}^{3}-96.6032 \hat{a}_{s}^{4} .
\end{aligned}
$$

The massive corrections start at $O\left(a_{s}^{0}\right)$ with the $s^{\prime}=\left(\left|V_{d c}\right|^{2} d+\left|V_{s c}\right|^{2} s\right) \rightarrow c$ transitions [6,7] and have been given in complete form in Ref. [15] to 2-loop order. The charm corrections at $O\left(\hat{a}_{s}^{2}\right)$ are of the same size as the massless $O\left(\hat{a}_{s}^{4}\right)$ corrections. Ref. [15] also contains the target mass corrections. In the asymptotic case, the effect of the heavy flavor corrections reduces to a shift of $N_{F} \rightarrow N_{F}+1$ in the massless corrections since the massive OMEs vanish for $N=1$ due to fermion conservation, which holds to all orders in perturbation theory.

\section{Conclusions}

We have calculated the massive charm quark 3-loop corrections to the charged current Wilson coefficients for the structure functions $F_{1,2}^{W^{+}-W^{-}}\left(x, Q^{2}\right)$ in the asymptotic region $Q^{2} \gg m_{c}^{2}$ both in Mellin $N$ and $x$ space. The corresponding contributions are composed of two massive Wilson coefficients $L_{q}^{W^{+}-W^{-}, \text {NS }}$ and $H_{q}^{W^{+}-W^{-}, \text {NS }}$ for which the weak boson either couples to a massless $(L)$ or a massive quark line $(H)$, here in the $s^{\prime} \rightarrow c$ transition. The massless 3-loop Wilson coefficients have been calculated in [3] and the massive OMEs were presented before in [19] as 
part of the present project to compute all massive 3-loop corrections to deep-inelastic scattering at high values of $Q^{2}$. The results have a representation in terms of nested harmonic sums and harmonic polylogarithms only. The charm quark corrections in case of both structure functions amount up to $\sim 8 \%$, depending on $x$ and the 3-loop corrections stabilize lower order QCD results. At low values of $Q^{2}$, effects of power corrections are still visible, which we have illustrated using recent complete 2-loop results [15], while for $Q^{2} \gtrsim 100 \mathrm{GeV}^{2}$ the asymptotic representation is valid in a rather wide range of $x$.

We also discussed potential contributions of the present corrections to the Adler and unpolarized Bjorken sum rules. In the former case, in accordance with the expectation, no corrections are obtained. For the Bjorken sum rule, the charm quark contributions lead to a shift of $N_{F}=3$ by one unit in the massless result. There are no heavy quark contributions due to fermion number conservation, which is expressed by a vanishing first moment of the operator matrix element in the non-singlet cases. Therefore, only the massless terms contribute now with $N_{F} \rightarrow N_{F}+1$.

The 3-loop charm quark corrections to the structure functions $F_{1,2}^{W^{+}-W^{-}}\left(x, Q^{2}\right)$ will improve the analysis of the HERA charged current data and are relevant for precision measurements in deep-inelastic scattering at planned facilities like the EIC [39], LHeC [40] and neutrino factories [41] in the future, which will reach a higher statistical and systematic precision than obtained in present experiments.

Acknowledgment. We would like to thank J. Ablinger, A. Hasselhuhn and A. Vogt for discussions, as well A. Vogt for providing us the effective numerical representations of the massless flavor non-singlet 3-loop Wilson coefficients of Ref. [3]. This work was supported in part by the European Commission through contract PITN-GA-2012-316704 (HIGGSTOOLS) and the Austrian Science Fund (FWF) grant SFB F50 (F5009-N15). 


\section{References}

[1] J.D. Bjorken, Phys. Rev. 163 (1967) 1767.

[2] S.L. Adler, Phys. Rev. 143 (1966) 1144.

[3] J. Davies, A. Vogt, S. Moch and J.A.M. Vermaseren, arXiv:1606.08907 [hep-ph], and in preparation.

[4] W.A. Bardeen, A.J. Buras, D.W. Duke and T. Muta, Phys. Rev. D 18 (1978) 3998.

[5] W. Furmanski and R. Petronzio, Z. Phys. C 11 (1982) 293 and references therein.

[6] J. Blümlein, A. Hasselhuhn, P. Kovacikova and S. Moch, Phys. Lett. B 700 (2011) 294 arXiv:1104.3449 [hep-ph]].

[7] M. Glück, S. Kretzer and E. Reya, Phys. Lett. B 398 (1997) 381 [Erratum: Phys. Lett. B 405 (1997) 392] [hep-ph/9701364].

[8] T. Gottschalk, Phys. Rev. D 23 (1981) 56.

[9] M. Buza and W.L. van Neerven, Nucl. Phys. B 500 (1997) 301 hep-ph/9702242.

[10] E.B. Zijlstra and W.L. van Neerven, Phys. Lett. B 297 (1992) 377.

[11] S. Moch and J.A.M. Vermaseren, Nucl. Phys. B 573 (2000) 853 hep-ph/9912355.

[12] J. Blümlein, A. Hasselhuhn and T. Pfoh, Nucl. Phys. B 881 (2014) 1 arXiv:1401.4352 [hep-ph]].

[13] A. Hasselhuhn, 3-Loop Contributions to Heavy Flavor Wilson Coefficients of Neutral and Charged Current DIS, PhD Thesis TU Dortmund, 2013, DESY-THESIS-2013-050.

[14] M. Buza, Y. Matiounine, J. Smith, R. Migneron and W. L. van Neerven, Nucl. Phys. B 472 (1996) 611 hep-ph/9601302.

[15] J. Blümlein, G. Falcioni and A. De Freitas, Nucl. Phys. B 910 (2016) 568 arXiv:1605.05541 [hep-ph]].

[16] A. Arbuzov, D.Y. Bardin, J. Blümlein, L. Kalinovskaya and T. Riemann, Comput. Phys. Commun. 94 (1996) 128 hep-ph/9511434];

J. Blümlein, M. Klein, T. Naumann and T. Riemann, PHE-88-01.

[17] J. Blümlein, Prog. Part. Nucl. Phys. 69 (2013) 28 arXiv:1208.6087 [hep-ph]].

[18] I. Bierenbaum, J. Blümlein and S. Klein, Nucl. Phys. B 820 (2009) 417 arXiv:0904.3563 [hep-ph]].

[19] J. Ablinger, A. Behring, J. Blümlein, A. De Freitas, A. Hasselhuhn, A. von Manteuffel, M. Round, C. Schneider, and F. Wißbrock, Nucl. Phys. B 886 (2014) 733 arXiv:1406.4654 [hep-ph]].

[20] N. Cabibbo, Phys. Rev. Lett. 10 (1963) 531.

[21] M. Kobayashi and T. Maskawa, Prog. Theor. Phys. 49 (1973) 652. 
[22] K.A. Olive et al. (Particle Data Group), Chin. Phys. C, 38, 090001 (2014).

[23] A. Behring, J. Blümlein, A. De Freitas, A. Hasselhuhn, A. von Manteuffel and C. Schneider, Phys. Rev. D 92 (2015) 11, 114005 [arXiv:1508.01449 [hep-ph]].

[24] A. Behring, J. Blümlein, A. De Freitas, A. von Manteuffel and C. Schneider, Nucl. Phys. B 897 (2015) 612 arXiv:1504.08217 [hep-ph]].

[25] J. Ablinger, A. Behring, J. Blümlein, G. Falcioni, A. De Freitas, A. Hasselhuhn, A. von Manteuffel, M. Round, C. Schneider, F. Wißbrock, PoS (LL2016) 065 arXiv:1609.03397 [hep-ph]];

J. Blümlein, A. DeFreitas and C. Schneider, Nucl. Part. Phys. Proc. 261-262 (2015) 185 arXiv:1411.5669 [hep-ph]].

[26] J.A.M. Vermaseren, Int. J. Mod. Phys. A 14 (1999) 2037 hep-ph/9806280];

J. Blümlein and S. Kurth, Phys. Rev. D 60 (1999) 014018 [hep-ph/9810241].

[27] E. Remiddi and J.A.M. Vermaseren, Int. J. Mod. Phys. A 15 (2000) 725 hep-ph/9905237.

[28] J. Blümlein, Comput. Phys. Commun. 159 (2004) 19 [hep-ph/0311046].

[29] S. Alekhin, J. Blümlein, K. Daum, K. Lipka and S. Moch, Phys. Lett. B 720 (2013) 172 arXiv:1212.2355 [hep-ph]].

[30] S. Alekhin, J. Blümlein and S. Moch, Phys. Rev. D 89 (2014) 054028 arXiv:1310.3059 [hep-ph]].

[31] V. Ravindran and W.L. van Neerven, Nucl. Phys. B 605 (2001) 517 [hep-ph/0102280|.

[32] S.L. Adler, arXiv:0905.2923 [hep-ph].

[33] S. Moch and M. Rogal, Nucl. Phys. B 782 (2007) 51 arXiv:0704.1740 [hep-ph]].

[34] G. Altarelli, R.K. Ellis and G. Martinelli, Nucl. Phys. B 143 (1978) 521, Erratum: Nucl. Phys. B 146 (1978) 544.

[35] B. Humpert and W.L. van Neerven, Nucl. Phys. B 184 (1981) 225.

[36] S.G. Gorishnii and S.A. Larin, Phys. Lett. B 172 (1986) 109.

[37] S.A. Larin, F.V. Tkachov and J.A.M. Vermaseren, Phys. Rev. Lett. 66 (1991) 862.

[38] K.G. Chetyrkin, Talk at Loops and Legs in Quantum Field Theory, Weimar, April 2014, https://indico.desy.de/conferenceOtherViews.py?view=standard\&conf $I d=8107$

[39] D. Boer, M. Diehl, R. Milner, R. Venugopalan, W. Vogelsang, D. Kaplan, H. Montgomery, S. Vigdor et al., arXiv:1108.1713 [nucl-th];

A. Accardi, J.L. Albacete, M. Anselmino, N. Armesto, E.C. Aschenauer, A. Bacchetta, D. Boer, W. Brooks et al., arXiv:1212.1701 [nucl-ex].

[40] J.L. Abelleira Fernandez et al. [LHeC Study Group Collaboration], J. Phys. G 39 (2012) 075001 arXiv:1206.2913 [physics.acc-ph]].

[41] D.M. Kaplan [MAP and MICE Collaborations], EPJ Web Conf. 95 (2015) 03019 arXiv:1412.3487 [physics.acc-ph]]. 